\title{
On the efficiency and reliability of cluster mass estimates based on member galaxies
}

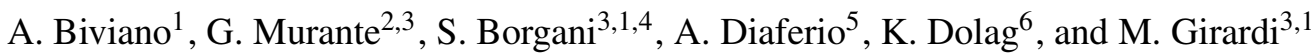 \\ 1 INAF/Osservatorio Astronomico di Trieste, via Tiepolo 11, 34131 Trieste, Italy \\ e-mail: biviano@oats.inaf.it \\ 2 INAF/Osservatorio Astronomico di Torino, Strada Osservatorio 20, 10025 Pino Torinese, Italy \\ Dipartimento di Astronomia dell'Università di Trieste, via Tiepolo 11, 34131 Trieste, Italy \\ INFN - National Institute for Nuclear Physics, Trieste, Italy \\ 5 Dipartimento di Fisica Generale “Amedeo Avogadro”, Universitá degli Studi di Torino, Torino, Italy \\ 6 Max-Planck-Institut für Astrophysik, Karl-Schwarzschild Strasse 1, Garching bei München, Germany
}

Received 26 January 2006 / Accepted 5 May 2006

ABSTRACT

\begin{abstract}
Aims. We study the efficiency and reliability of cluster mass estimators that are based on the projected phase-space distribution of galaxies in a cluster region.

Methods. We analyse a data-set of 62 clusters extracted from a concordance $\Lambda \mathrm{CDM}$ cosmological hydrodynamical simulation. We consider both dark matter (DM) particles and simulated galaxies as tracers of the clusters gravitational potential. Two cluster mass estimators are considered: the virial mass estimator, corrected for the surface-pressure term, and a mass estimator (that we call $M_{\sigma}$ ) based entirely on the velocity dispersion estimate of the cluster. In order to simulate observations, galaxies (or DM particles) are first selected in cylinders of given radius (from 0.5 to $1.5 h^{-1} \mathrm{Mpc}$ ) and $\simeq 200 h^{-1} \mathrm{Mpc}$ length. Cluster members are then identified by applying a suitable interloper removal algorithm.

Results. The virial mass estimator overestimates the true mass by $\simeq 10 \%$ on average, for sample sizes of $\gtrsim 60$ cluster members. For similar sample sizes, $M_{\sigma}$ underestimates the true mass by $\simeq 15 \%$, on average. For smaller sample sizes, the bias of the virial mass estimator substantially increases, while the $M_{\sigma}$ estimator becomes essentially unbiased. The dispersion of both mass estimates increases by a factor $\sim 2$ as the number of cluster members decreases from $\sim 400$ to $\sim 20$.

It is possible to reduce the bias in the virial mass estimates either by removing clusters with significant evidence for subclustering or by selecting early-type galaxies, which substantially reduces the interloper contamination. Early-type galaxies cannot however be used to improve the $M_{\sigma}$ estimates since their intrinsic velocity distribution is slightly biased relative to that of the DM particles.

Radially-dependent incompleteness can drastically affect the virial mass estimates, but leaves the $M_{\sigma}$ estimates almost unaffected. Other observational effects, like centering and velocity errors and different observational apertures, have little effect on the mass estimates.
\end{abstract}

Key words. galaxies: clusters: general - galaxies: kinematics and dynamics - cosmology: observations methods: $N$-body simulations

\section{Introduction}

The masses of galaxy clusters are a very useful observable in cosmology. The number density of clusters of galaxies above a given mass threshold, and the evolution of this abundance with redshift $z$, provide in principle strong constraints on cosmological models/parameters (see, e.g., Rosati et al. 2002; Voit 2005, for recent reviews, and references therein). The advantage of using galaxy clusters is that they are massive, luminous objects, that can be detected relatively easily out to $z \gtrsim 1$ by several techniques in different wavebands. The disadvantage is that they are rather complex objects, hence their masses are not easily estimated, and can often be plagued by systematic effects that are not easy to correct for.

Since Zwicky's (1933) first estimate of a cluster mass, based on the application of the virial theorem to the projected phasespace distribution of galaxies in the Coma cluster, cluster mass determinations have always been taken with some caution (see the historical review of Biviano 2000). A cluster mass estimate based on the observed projected phase-space distribution of galaxies can be wrong because of several effects. Quite important in this respect are the projection effects leading to the inclusion of interlopers in the sample of presumed cluster members (see, e.g., Lucey 1983; Borgani et al. 1997; Cen 1997, C97 hereafter). Biases in the cluster mass estimates can also occur when the studied cluster is far from virialization, e.g. during the accretion phase of a massive group (see, e.g. Girardi \& Biviano 2002), or if galaxies are biased tracers of the gravitational potential, which could happen as a consequence of dynamical friction (e.g. Biviano et al. 1992; Goto 2005), or as a consequence of infalling motions (e.g., Moss \& Dickens 1977; Biviano et al. 1997).

Because of the abovementioned problems, other methods of cluster mass determination have been considered that are not based on the phase-space distribution of galaxies. While lensing mass estimates are also known to be affected by projection effects, these are generally believed to be less severe (e.g. Reblinsky \& Bartelmann 1999, RB99 hereafter; Clowe et al. 2004; but see Metzler et al. 1999), unless the cluster is elongated or has substantial substructure along the line-of-sight 
(Athreya et al. 2002; Bartelmann \& Steinmetz 1996; Gavazzi 2005; Oguri et al. 2005). Projection effects are even less important in the case of X-ray emission-based cluster mass estimates, since the X-ray emissivity is proportional to the square of the gas density.

Problems with lensing and X-ray mass estimates do exist, however. Masses determined with the lensing technique are affected by the mass-sheet degeneracy that cannot always be broken (e.g. Dye et al. 2001; Cypriano et al. 2004). The effect of intervening matter along the line-of-sight is to increase the weak-lensing mass estimates of clusters, especially at high redshifts (Metzler et al. 2001; Lombardi et al. 2005; Wambsganss et al. 2004, 2005). Lensing and X-ray-based mass estimates have often been found to be discordant, and this is generally interpreted as evidence of non-equilibrium (e.g. Wu 2000; Clowe et al. 2001; Athreya et al. 2002; Smith et al. 2002; Cypriano et al. 2004; Ota et al. 2004; Bradač et al. 2005). In clusters undergoing merging events the X-ray luminosity and temperature can be boosted, thus leading to an overestimate of the cluster mass (e.g. Schindler \& Müller 1993; Ricker \& Sarazin 2001; Barrena et al. 2002; Diaferio et al. 2005). On the other hand, violations of the condition of hydrostatic equilibrium, inaccurate modelling of the gas density profile and observational biases in the measure of the intra-cluster gas temperature, may lead to a sizeable underestimate of the cluster mass (e.g. Bartelmann \& Steinmetz 1996; Kay et al. 2004; Rasia et al. 2004).

A combination of several, independent cluster mass estimates is likely to provide the most accurate results. Moreover, with the Sloan Digital Sky Survey coming to completion (e.g. Abazajian et al. 2005), a large number of nearby clusters with $\geq 30$ galaxies with redshifts is now available (Miller et al. 2005). For many of these clusters, X-ray data are not available, and mass estimates must be based on optical data. Hence, mass estimates based on the projected phase-space distribution of galaxies are still very useful.

It is thus appropriate to re-assess the reliability of mass estimates of clusters based on the dynamics of their member galaxies. Previous analyses have generally considered only specific aspects of this topic. Initially, the reliability of different mass estimators has been assessed from $N$-body simulations outside of a cosmological context (Danese et al. 1981; Perea et al. 1990; Aceves \& Perea 1999). In order to properly deal with this topic, clusters must however be identified within cosmological simulations with large box-sizes. This has been achieved by several studies (e.g. Frenk et al. 1990; Borgani et al. 1997; C97; van Haarlem et al. 1997, hereafter vH97; RB99; Sanchis et al. 2004; Łokas et al. 2005), where, however, dark matter (DM hereafter) particles and not galaxies were considered as tracers of the potential. Van Kampen \& Katgert (1997) used the method of constrained random fields to increase the numerical resolution of their simulation, but by doing this they were unable to consider projection effects. In most analyses, interlopers were rejected using Yahil \& Vidal's (1977) traditional 3- $\sigma$-clipping method (but see, e.g., vH97 who also tested the more sophisticated method of den Hartog \& Katgert 1996; and Sanchis et al. 2004; and Łokas et al. 2005, who tested their own methods of interloper removal).

A general conclusion from these studies is that cluster mass estimates can be severely affected by projection effects. This happens mainly as a consequence of the cluster identification process in $2 \mathrm{~d}$ projected space, performed with Abell's (1958) original algorithm. Frenk et al. (1990) argued that cluster masses are systematically over-estimated, but subsequent studies (C97; vH97; RB99) concluded that cluster masses can be either over- or under-estimated, depending on the projection angle, the cluster mass, and the algorithm used to remove interlopers. Sanchis et al. (2004) and Łokas et al. (2005) found a rather good agreement between estimated and true cluster masses, when a large number of tracers of the potential was considered in each cluster.

None of the abovementioned studies tried to identify galaxies in the cosmological simulations. In those studies where this was achieved, it was generally found that the spatial distribution of subhaloes, selected by their mass, is less concentrated than that of DM (e.g. Ghigna et al. 1998; Klypin et al. 1999; Gao et al. 2004). However, when gas dynamics was included in the simulations, galaxies, selected by luminosity, turned out to have a considerably more concentrated spatial distribution than subhaloes, and more similar to that of DM (Berlind et al. 2003; Gao et al. 2004; Nagai \& Kravtsov 2005). This occurs because tidal stripping induce substantial mass loss from galaxy haloes, but very little stellar mass loss.

As far as the velocity distribution of subhaloes is concerned, most studies have found it to be wider than that of DM particles (Colín et al. 2000; Diemand et al. 2004), at least near the cluster centre (Ghigna et al. 2000; Reed et al. 2005). Based on hydrodynamical simulations, Frenk et al. (1996) concluded that galaxies suffer significant dynamical friction, and are slowed down relative to DM particles, to such an extent that cluster mass estimates based on the velocity dispersion of galaxies are likely to be in error by factors of $0.25-0.75$, depending on the masses of the galaxies selected as tracers. On the other hand, Berlind et al. (2003) found that galaxies have only a mild velocity bias with respect to DM, and Faltenbacher et al. (2005) found that galaxies, if anything, move slightly faster than DM particles.

In this paper the reliability of cluster mass estimates based on the dynamics of their member galaxies is reconsidered on the basis of a set of clusters extracted from a large cosmological hydrodynamical simulation (Borgani et al. 2004; see also Murante et al. 2004), performed using the TREE+SPH GADGET-2 code (Springel et al. 2001; Springel 2005), for a concordance $\Lambda \mathrm{CDM}$ model. This simulation samples a fairly large volume (box size of $192 h^{-1} \mathrm{Mpc}$, where $h$ is the Hubble constant in units of $100 \mathrm{~km} \mathrm{~s}^{-1} \mathrm{Mpc}^{-1}$ ) which contains more than 100 clusters with mass above $10^{14} h^{-1} M_{\odot}$, while also having good enough resolution $\left(m_{\mathrm{DM}}=4.62 \times 10^{9} h^{-1} M_{\odot}\right.$ for the mass of the DM particles) to allow resolving halos hosting bright galaxies. Finally, the inclusion of the processes of radiative cooling, star formation and supernova feedback allows us to have a realistic description of the gas evolution and of the galaxy formation process.

In this paper we address the question of how accurate cluster mass determinations are, based on the dynamics of their member galaxies, under a variety of observational conditions, and for a wide range of cluster masses, but independently of the clusters identification procedure. No attempt is made here to simulate the observational identification algorithms of galaxy clusters, such as Abell's (1958) original one. In this sense, our approach is different from previous ones (Frenk et al. 1990; C97; vH97; RB99), in that we disentangle the problem of cluster mass estimation from that of cluster identification. The rationale for this choice is that today there is no standard cluster identification algorithm. Automated scans of digitized plates have since replaced Abell's eyeball identification of galaxy clusters (Dalton et al. 1992; Lumsden et al. 1992; Lopes et al. 2004), and much more sophisticated algorithms than Abell's (1958) have been applied to extract the 2-dimensional signal produced by a galaxy overdensity (e.g. Ramella et al. 2001; Gladders \& Yee 2005, and 
references therein). Moreover, those clusters whose masses are derived using member galaxies are not necessarily optically selected (e.g. Popesso et al. 2005). The results of this paper are therefore useful for a better understanding of why different techniques (lensing, X-ray, galaxies) can lead to discrepant cluster mass estimates, and also for the study of scaling relations of different cluster properties with cluster masses. Translating the results of this paper to the study of the distribution of observed cluster masses in a given survey requires convolution with the selection function of the survey itself.

The plan of this paper is as follows. In Sect. 2 the set of simulated clusters and its characteristics are described. In Sect. 3 the steps involved in the determination of cluster dynamical quantities are briefly described. The analyses of the cluster dynamics in projected phase-space are described in Sect. 4. Results are discussed and summarized in Sect. 5. In Sect. 6 we provide our conclusions.

\section{The simulated clusters}

The set of simulated clusters analysed in this paper are extracted from a large cosmological hydrodynamical simulation presented by Borgani et al. (2004), and performed using the massively parallel Tree+SPH GADGET-2 code (Springel et al. 2001; Springel 2005). We refer to Borgani et al. (2004) for a complete description of this simulation and of the cluster identification procedure. We provide here their main characteristics and describe in some detail the galaxy identification procedure. The simulation assumes a cosmological model with $\Omega_{0}=0.3$, $\Omega_{\Lambda}=0.7, \Omega_{\mathrm{b}}=0.019 h^{-2}, h=0.7$, and $\sigma_{8}=0.8$. The box size is $192 h^{-1} \mathrm{Mpc}$. We used $480^{3} \mathrm{DM}$ particles and (initially) as many gas particles, for a mass resolution of $m_{\mathrm{DM}}=4.62 \times$ $10^{9} h^{-1} M_{\odot}$. The Plummer-equivalent softening length was set to $\epsilon=7.5 h^{-1} \mathrm{kpc}$ at $z=0$. The simulation code includes explicit energy end entropy conservation, radiative cooling, a uniform time-dependent UV background (Haardt \& Madau 1996), the self-regulated hybrid multi-phase model for star formation (Springel \& Hernquist 2003), and a phenomenological model for galactic winds powered by type-II supernovae.

We identify galaxy clusters at redshift $z=0$ by applying a standard Friends-of-friends (FoF) analysis to the DM particle set, with linking length $\lambda=0.15$ in units of the mean interparticle distance. After the FoF identification, we applied a spherical overdensity criterion to find the virial region of each cluster (corresponding to an overdensity of $\approx 100$ times the critical density for the adopted cosmology). We identify 117 galaxy clusters whose virial mass is larger than $10^{14} h^{-1} M_{\odot}$ within our simulated volume. A subset of these clusters is selected for the analysis discussed in the following. The position of the minimum potential particle of each FoF group is then used as the centre for a spherical overdensity algorithm, which identifies the radii encompassing different overdensities.

Galaxies are identified using the publicly available algorithm $^{1}$ SKID (Stadel 2001). SKID calculates a density field using all the particles. Density is assigned with a spline-kernel similar to that employed by SPH codes; an important parameter is the number of neighbouring particles $N_{\text {sm }}$ on which the density is softened. Particles are then associated with the local maxima of such a field, which should represent the positions of the substructure. To this end, particles are "moved" along the gradient of the field until they begin to oscillate around the peaks and are then

1 See http://www-hpcc. astro. washington. edu/tools/ skid.html grouped using a FoF analysis on the "moved" positions. An unbinding procedure is applied to the resulting FoF groups, to discard particles that are not gravitationally bound to the group. All particles within a sphere of given radius are used to evaluate the gravitational potential. This step is performed on the "true" (i.e. not "moved") positions. All the length scales involved in the process are set starting from the parameter $\tau$ which represents the typical size of the objects to be found. Apart from the evaluation of the density field, it is possible to select to which types of particles (DM, stars, and gas) the algorithm must be applied. We used only star particles to identify our galaxies.

The problem of reliably detecting sub-structures within given DM haloes is long-lasting and still not uniquely solved. It is outside the purpose of the present work to discuss such an issue. We performed a number of tests on the SKID galaxies, to verify that the least possible number of objects is missed by the analysis and that the identified object are real ones. Such tests are presented elsewhere (Murante et al., in preparation). Our galaxies have been identified by using $\tau=20 h^{-1} \mathrm{kpc}$, which, after a trial-and-error procedure, turned out to be the optimal choice. This choice is also motivated by the fact that $20 h^{-1} \mathrm{kpc}$ roughly corresponds to the effective force resolution of the simulation. We also determined that distinct SKID analyses are needed, with different values of $N_{\text {sm }}$, that we assumed to be 16, 32 and 64. Some galaxies are "missed" using only one value for $N_{\mathrm{sm}}$. Therefore, we built our catalogue by combining together the results of the three SKID analyses, with the following rule: if a star particle is a member of a galaxy for one value of $N_{\mathrm{sm}}$, it is considered to belong to that galaxy; if a particle is member of two different galaxies for two different values of $N_{\mathrm{sm}}$, the two galaxies are then merged into a single object. SKID objects with less than 32 particles are discarded.

For the purpose of this paper it is not useful to consider clusters with less than 10 galaxies, hence in the following we restrict our analysis to those 62 clusters with at least 10 galaxies within a sphere of "virial" radius $r_{\mathrm{v}}$, defined as the radius where the mass density of the cluster equals 200 times the critical density of the Universe.

In Borgani et al. (2004) we have shown that these simulated clusters have on average a star fraction which is $\sim 50$ per cent larger than the typical observed values (Lin et al. 2003). However, this overcooling is mainly contributed by the central galaxy. Therefore, our simulations are expected to reliably describe the way in which cluster galaxies trace the underlying cluster dynamics.

For each galaxy, we define its formation redshift, $z_{\mathrm{f}}$, as the average value of the formation redshifts of all its member star particles. The value of $z_{\mathrm{f}}$ is also used to classify our galaxies into "early-type" and "late-type". Specifically, we consider a galaxy to be of early-type if $z_{\mathrm{f}} \geq 1.25$, and of late-type if $z_{\mathrm{f}}<1.25$. This choice is admittedly rather crude and is based on the comparison of our simulated galaxy sample with the sample of cluster galaxies of the ESO Nearby Abell Clusters Survey (ENACS, Katgert et al. 1996, 1998). In the ENACS, $64 \%$ of the galaxies identified as cluster members within $1.5 r_{\mathrm{v}}$ are classified as early-type galaxies (ellipticals, lenticulars, or an intermediate class between these two; see Biviano et al. 2002; Thomas \& Katgert 2006). Similarly, in the simulated clusters, $64 \%$ of the galaxies within $1.5 r_{\mathrm{v}}$ have $z_{\mathrm{f}} \geq 1.25$.

Further support for the identification of $z_{\mathrm{f}} \geq 1.25$ galaxies as early-type galaxies comes from the comparison of their distribution in projected phase-space with that observed for the ENACS cluster galaxies. Since the number of galaxies per simulated cluster is rather limited, clusters are stacked together by 


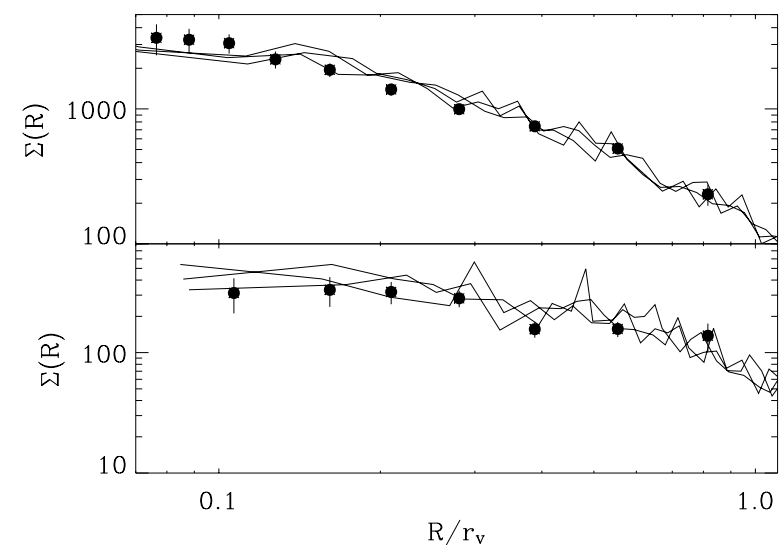

Fig. 1. The projected number density profiles of simulated galaxies (solid lines) and real galaxies from the ENACS sample (dots with error bars). The three solid lines in each plot correspond to three orthogonal projections. The normalisations of the simulated galaxies profiles have been arbitrarily scaled to match the observed profiles. The clustercentric distances on the $x$-axis are in units of the cluster virial radius. Top panel: simulated galaxies with formation redshift $z_{\mathrm{f}} \geq 1.25$ (see text) vs. earlytype galaxies from the ENACS sample. Bottom panel: simulated galaxies with $z_{\mathrm{f}}<1.25 \mathrm{vs}$. late-type galaxies from the ENACS sample.

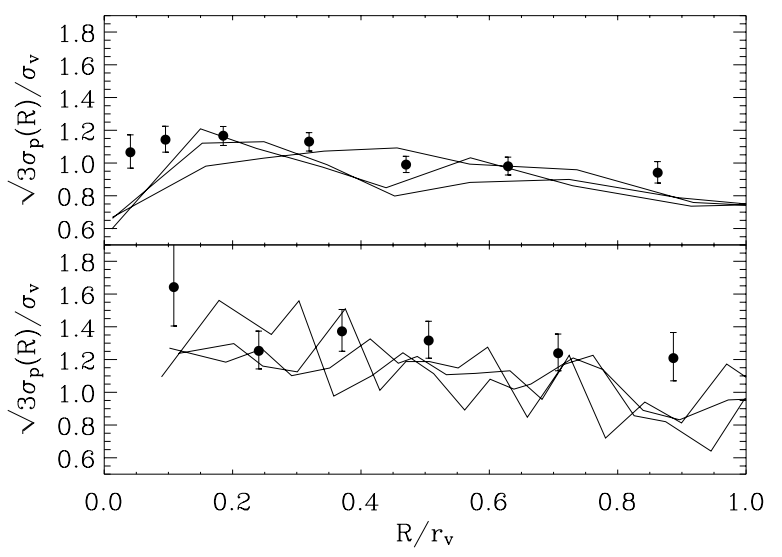

Fig. 2. The line-of-sight velocity dispersion profiles (in units of the total cluster velocity dispersion) of simulated galaxies (solid lines) and real galaxies from the ENACS sample (dots with error bars). The clustercentric distances on the $x$-axis are in units of the cluster virial radius. The three solid lines in each plot correspond to three orthogonal projections. Top panel: simulated galaxies with formation redshift $z_{\mathrm{f}} \geq 1.25$ (see text) vs. early-type galaxies from the ENACS sample. Bottom panel: simulated galaxies with $z_{\mathrm{f}}<1.25$ vs. late-type galaxies from the ENACS sample.

scaling the galaxy clustercentric distances and velocities by the value of $r_{\mathrm{v}}$ and the velocity dispersion of the cluster they belong to. A similar procedure is applied to the observed galaxies (see Katgert et al. 2004; Biviano \& Katgert 2004). The projected number density profiles, $\Sigma(R)$, and the line-of-sight velocity dispersion profiles, $\sigma_{\mathrm{p}}(R)$, of real and simulated galaxies are shown in Figs. 1 and 2, respectively, separately for early- and late-type galaxies (top and bottom panels, respectively). The simulated galaxies $\Sigma(R)$ s have been arbitrarily rescaled to match the observed $\Sigma(R)$ s, since here we are only interested in the relative distributions of simulated and real galaxies.

The profiles of observed and simulated galaxies are rather similar, both for the early- and the late-types. The main difference between the observed and simulated profiles is the lower number density and smaller velocity dispersion of the simulated early-type galaxies in the inner regions $\left(R / r_{\mathrm{v}} \lesssim 0.1\right)$. This difference is related to the well-known overmerging problem which affects the simulations in the denser regions. While this can be a source of concern when comparing simulated vs. observed cluster mass profiles, we argue that the analysis of cluster masses is not significantly influenced by this problem. In fact, the apparent underdensity of simulated early-type galaxies in the inner regions, as compared to the real ones, is not significant. The fraction of early-type galaxies within the virial region that lie within $0.1 r_{\mathrm{v}}$ is $12.9 \%$ for the simulated galaxies and $14.5 \%$ for the observed ones. As a consequence, no significant effect is expected on cluster harmonic mean radius estimates (which enters the virial mass estimate, see Sect. 3), also because these fractions are low. For the same reason, no significant effect is expected on cluster global velocity dispersion estimates, despite the fact that the difference in the velocity dispersions of simulated and observed early-type galaxies is significant within $0.1 r_{\mathrm{v}}$. In fact, the ratio between the velocity dispersion estimates obtained using all galaxies and only those with $R>0.1 r_{\mathrm{v}}$ is 0.994 for the simulated galaxies and 1.008 for the real ones.

We conclude that the similarity between the profiles of simulated and observed galaxies lends support to our choice of $z_{\mathrm{f}}=1.25$ for separating early- and late-type galaxies in our simulations.

\section{The cluster mass estimates}

Several different definitions of "virial mass" have been given in the literature. This term may be used to define the total mass of a cluster within a radius of given overdensity, i.e. the "virial radius", typically the radius where the mean cluster mass density equals 100 or 200 times the Universe critical density (see, e.g., Łokas \& Mamon 2003). On the other hand, the same term is used to define the mass estimated through application of the virial theorem to the cluster galaxies within an observationally defined aperture (e.g. Biviano et al. 1993; Girardi et al. 1998). This estimate requires not only an estimate of the cluster velocity dispersion, but also of the harmonic mean radius of the spatial distribution of cluster galaxies. To further complicate the issue, twice the harmonic mean radius is also usually referred to as the "virial radius" (e.g. Girardi et al. 1998; Merchán \& Zandivarez 2005).

The virial mass estimate requires correction for the surface pressure term (The \& White 1986), unless the entire system is contained within the observationally defined aperture radius (see also Macciò et al. 2003). Unfortunately, it is still relatively uncommon to see this correction applied (see, e.g., Koranyi et al. 1998). Neglecting this correction leads to overestimate the mass of a system (see, e.g., Carlberg et al. 1997a), and this can partly account for some of the claimed discrepancies between optically and X-ray derived cluster mass estimates.

For the sake of clarity, we detail in the following the procedure that we apply to our simulated clusters in projection in order to simulate an observational estimate of their masses. Such a procedure has recently been applied to a large set of nearby clusters by Popesso et al. (2005, 2006).

We define a cluster "true" mass, $M_{\mathrm{v}}$, as the total mass within the radius $r_{\mathrm{v}}$, where the mass density of the cluster equals 200 times the critical density of the Universe at the cluster redshift (we call $\sigma_{\mathrm{v}}$ the $3 \mathrm{~d}$ velocity dispersion of the DM particles within the same radius). The virial mass estimate $\tilde{M}_{\mathrm{v}}$ within the same radius is computed as follows. 
1. Define an aperture radius, $a$, within which to perform the dynamical analysis, and select all DM particles or all galaxies within a cylinder of radius $a$ and $\simeq 200 h^{-1} \mathrm{Mpc}$ length, along each of three orthogonal projections. Cluster centres are computed as the positions of the minimum potential particle in each cluster (see Sect. 2), unless otherwise indicated (see Sect. 4.5). In virtually all cases, these centres correspond (to within $\sim 10 \mathrm{kpc}$ ) with the peaks of the cluster X-ray emissivity. The same centre choice is usually adopted observationally, when X-ray data are available (see, e.g., Biviano et al. 1997; Popesso et al. 2005).

2. Select cluster members. This is done first by using a cut in line-of-sight velocity space of $\pm 4000 \mathrm{~km} \mathrm{~s}^{-1}$ with respect to the mean cluster velocity, initially defined by applying the biweight estimator ${ }^{2}$ (see Beers et al. 1990) to all the objects within $0.5 h^{-1} \mathrm{Mpc}$ from the cluster centre. In a second step, the weighted gap procedure of Girardi et al. (1993) is applied on the remaining objects. To the objects that pass the weighted gap selection we apply Katgert et al.'s (2004) procedure that makes use of the location of galaxies (or particles) in projected phase-space (see also den Hartog \& Katgert's 1996).

3. Determine the "robust" estimate of the line-of-sight velocity dispersion $\sigma_{a, p}$, within the aperture $a$, using the biweight or gapper estimator, depending on the number of data available, $\geq 15$ or $<15$, respectively (see Beers et al. 1990).

4. Correct the velocity dispersion estimate for velocity errors (if these are added to the simulated data, see Sect. 4.5) following the prescriptions of Danese et al. (1980).

5. Determine the projected harmonic mean radius $r_{a, p}$, within the aperture $a$.

6. Obtain a first estimate of the mass from

$$
M_{a} \equiv 3 \pi \sigma_{a, p}^{2} r_{a, p} / G
$$

where $G$ is the gravitational constant, the factor $3 \pi / 2$ is the deprojection factor, see Limber \& Mathews (1960), and a factor 2 is needed to convert the harmonic mean radius into the "virial radius" of Girardi et al. (1998).

7. Estimate the Navarro et al. (1997, NFW hereafter) concentration parameter using the relation $c=4\left(\sigma_{a, p} / 700\right)^{-0.306}$. The normalization of the relation is taken from Katgert et al. (2004), and the exponent of the relation is derived from Dolag et al. (2004), under the assumption that the mass scales with the third power of the velocity dispersion.

8. Correct the mass estimate for the surface pressure term, $f_{\mathrm{sp}}$, obtained for a NFW profile with concentration $c: M_{a, c} \equiv$ $f_{\mathrm{sp}} M_{a}$, assuming isotropic orbits (see Eq. (8) in Girardi et al. 1998). Note that the value of the correction factor is not negligible $\left(f_{\mathrm{sp}}=0.84\right.$ on average for our simulated clusters observed out to an aperture radius $\left.a=1.5 h^{-1} \mathrm{Mpc}\right)$.

9. Determine $\tilde{r}_{\mathrm{v}}$, an estimate of $r_{\mathrm{v}}$, as $\tilde{r}_{\mathrm{v}}=a\left[\rho_{a} /\left(200 \rho_{c}(z)\right)\right]^{1 / \xi}$, where $\rho_{c}(z)$ is the critical density of the Universe at the cluster redshift, $\rho_{a} \equiv M_{a, c} /\left(4 \pi a^{3} / 3\right)$, and $\xi$ is the local slope of a NFW profile of concentration $c$ at the radius $a$.

10. Determine $\tilde{M}_{\mathrm{v}}$, an estimate of $M_{\mathrm{v}}$, by extrapolating or interpolating $M_{a, c}$ from $a$ to $\tilde{r}_{\mathrm{v}}$ using a NFW profile with concentration $c$.

Quite often, cosmological constraints have been obtained directly from the distribution of cluster velocity dispersions, used as proxies for the cluster masses (see, e.g., Girardi et al. 1993).

${ }^{2}$ Here and throughout this paper we use the biweight estimator for the average and dispersion, unless otherwise indicated.

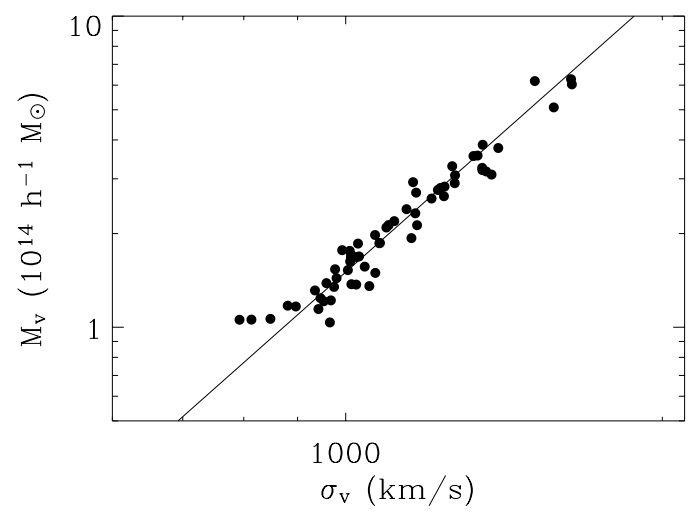

Fig. 3. The true mass vs. the $3 d$ velocity dispersion (both computed within a sphere of radius $r_{\mathrm{v}}$ ) for the 62 simulated clusters with at least 10 galaxies within $r_{\mathrm{v}}$. The best fitting cubic law relation is shown (solid line).

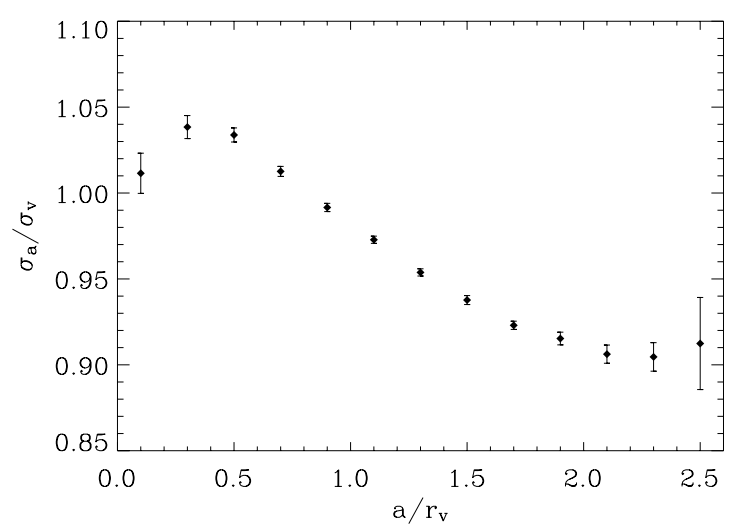

Fig. 4. The average integrated velocity dispersion profile for the 62 simulated clusters. The $3 \mathrm{~d}$ velocity dispersion within a sphere of radius $a$ is shown as a function of $a / r_{\mathrm{v}}$, in units of $\sigma_{\mathrm{v}}$. 1- $\sigma$ error bars are shown.

It is therefore useful to also consider an alternative (and simpler) mass estimator entirely based on the line-of-sight estimate of the velocity dispersion within a given aperture, $\sigma_{a, p}$. The relation between $M_{\mathrm{v}}$ and $\sigma_{\mathrm{v}}$ for our simulated clusters is shown in Fig. 3. The line in that plot represents the best-fitting cubic relation

$M_{\mathrm{v}} \equiv A\left(\frac{\sigma_{\mathrm{v}}}{10^{3} \mathrm{~km} \mathrm{~s}^{-1}}\right)^{3} \times 10^{14} h^{-1} M_{\odot}$

where $A=1.50 \pm 0.02(1-\sigma$ error $)$. We note that this relation is approximately independent of the cosmological model (Borgani et al. 1999). It is possible to use this relation to obtain a mass estimate entirely based on the velocity dispersion estimate. Since $\sqrt{3} \sigma_{a, p}$ can differ significantly from $\sigma_{\mathrm{v}}$ depending on the aperture radius $a$ chosen by the observer, we need to apply a correction that depends on the ratio $a / r_{\mathrm{v}}$. For each of the 62 clusters, we determine the 3-d velocity dispersion $\sigma_{a}$, within a sphere of radius $a$, for different values of $a$. The average $\sigma_{a} / \sigma_{\mathrm{v}}$ vs. $a / r_{\mathrm{v}}$ profile of our 62 clusters is shown in Fig. 4.

Hence, given a line-of-sight velocity dispersion estimate within a given observational aperture, $\sigma_{a, p}$, we proceed as follows. We use $\sqrt{3} \sigma_{a, p}$ to determine an initial estimate of the mass of the system through Eq. (2). We then obtain an estimate $\tilde{r}_{\mathrm{v}}$ of $r_{\mathrm{v}}$, following steps 7 and 9 above (note that step 8 is not needed in this case, because the relation provided by Eq. (2) relates the velocity dispersion to the true cluster mass, for which no surface term correction is needed). Using the $\sigma_{a} / \sigma_{\mathrm{v}}$ vs. $a / r_{\mathrm{v}}$ profile of Fig. 4, and replacing the true quantities $r_{\mathrm{v}}$ and $\sigma_{a}$, with 


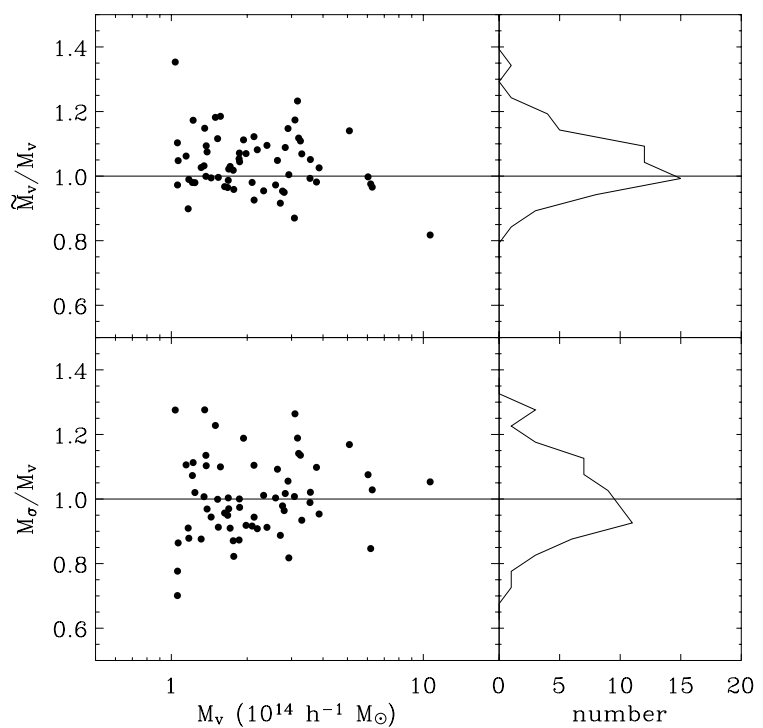

Fig. 5. Verifying the performance of the mass estimators: the comparison between the masses estimated using full phase-space information, and the true cluster masses. Top-left panel: ratio between the virial and true mass, $\tilde{M}_{\mathrm{v}} / M_{\mathrm{v}}$ vs. the true mass, $M_{\mathrm{v}}$. Bottom-left panel: ratio between the mass obtained from $\sigma_{\mathrm{v}}$ and the true mass, $M_{\sigma} / M_{\mathrm{v}}$ vs. the true mass $M_{\mathrm{v}}$. Top-right panel: distribution of $\tilde{M}_{\mathrm{v}} / M_{\mathrm{v}}$. Bottom-right panel: distribution of $M_{\sigma} / M_{\mathrm{v}}$. The masses $\tilde{M}_{\mathrm{v}}$ and $M_{\sigma}$ are estimated from the full phase-space distributions of $5000 \mathrm{DM}$ particles per system (when available; otherwise all DM particles are selected) within the cluster virial radius.

their estimates $\tilde{r}_{\mathrm{v}}$ and $\sqrt{3} \sigma_{a, p}$, respectively, we obtain an estimate of $\sigma_{\mathrm{v}}$ which we use to determine the $M_{\sigma}$ estimate of $M_{\mathrm{v}}$ through Eq. (2).

In order to verify the performance of these two mass estimators, we have applied them to the clusters in the simulation, making use of the full phase-space information. In this case, there is no need to apply the interloper-rejection procedure outlined above (step 2), since we randomly select 5000 DM particles in each cluster within a sphere (not a cylinder) of radius $a \equiv r_{\mathrm{v}}$ (when this sphere contains less than $5000 \mathrm{DM}$ particles, all particles are selected). Also the de-projection factor $3 \pi / 2$ (step 6 ) is not needed. As for the estimate of $M_{\sigma}$, we directly apply Eq. (2). In Fig. 5 we show the ratio between the virial $\left(\tilde{M}_{\mathrm{v}}\right)$ and the true masses (upper panel), and $M_{\sigma}$ and the true masses (lower panel) for our sample of 62 clusters. On average we find $\tilde{M}_{\mathrm{v}} / M_{\mathrm{v}}=1.03$ with a dispersion of 0.09 , and $M_{\sigma} / M_{\mathrm{v}}=1.00$ with a dispersion of 0.12 (see also Tormen et al. 1997, for a similar analysis). Perfect identity is not expected, since clusters are not fully virialized, but the good agreement indicates that the estimators are unbiased and the clusters in the simulation are close to virialization within $r_{\mathrm{v}}$, as expected for $z \sim 0$ clusters in a $\Lambda$ CDM cosmology.

\section{Dynamical analysis in projected phase-space}

In order to mimic observations, the simulated clusters are placed at a distance of $250 \mathrm{~h}^{-1} \mathrm{Mpc}$ from the hypothetical observer. Three orthogonal projections are considered for each cluster, leading to a total of $186(62 \times 3)$ cluster projections (except when we remove the cases with significant evidence for subclustering, see Sect. 4.2). DM particles or galaxies are then selected within cylinders of given radius, chosen to be a fraction (from $1 / 3$ to 1) of an Abell radius. This is meant to reproduce typical observational procedures where the virial radius of the system is unknown prior to observation. The length of the cylinder is set by the simulation cube.

In the following we analyse how cluster mass estimates are affected by several observational effects. In particular, we consider the effects of different sample sizes, different observational apertures, observational errors, incompleteness, and subclusters detected in projected phase-space. In principle, it would be desirable to perform all these analyses using the simulated galaxies as tracers of the potential, rather than the DM particles. As a matter of fact, the analyses performed on the simulated galaxies are likely to provide a reliable picture of the real observational situation, since the phase-space distributions of simulated galaxies are not too different from the observed ones (see Sect. 2). On the other hand, the DM particles are not distributed like the galaxies in the simulated clusters (see Sect. 4.6). Unfortunately, however, there are only few galaxies per cluster in our simulated set, hence small-number statistical noise becomes a dominant source of scatter. In order to overcome this problem, we have also considered DM particles as tracers of the gravitational potential, even if, of course, these are not true "observables".

The most relevant results of the dynamical analyses in projected phase-space are summarized in Table 1. The different analyses performed are identified by their number in the 1 st column of Table 1. Column 2 lists the observational aperture size, $a$, in $h^{-1} \mathrm{Mpc}$, i.e. the radius of the cylinder within which particles or galaxies are selected. Column 3 lists the number of DM particles per projection initially selected for the analysis within a cylinder of given radius. In the case of galaxies, all galaxies (of the specified type) are always selected, and this number changes from projection to projection, hence we list the average over all projections. This is also the case for DM particles, when we select an identical number of DM particles as of available galaxies within the cylinder (see Sect. 4.6). In Col. 4 "DM" or "G" indicates whether DM particles or Galaxies are chosen as tracers of the potential. When only galaxies of a given type are selected, this is further specified after the letter " $G$ ". Column 5 lists the type of selection performed on DM particles, which can either be random ("Rdm") or of a type that reproduces a single-mask, multi-slit observation ("Slit"). In the case of galaxies, "All" indicates that all galaxies in the cylinder are selected. The note "projections with subclusters excluded" indicates that the definition of the sample used is the same as in the line above, except that cluster projections with significant evidence of substructure have been removed from the sample. Columns 6 and 7 list the average of the number of DM particles or galaxies selected as cluster members within the chosen aperture, and the fraction $f_{\text {out }}$ of these that actually lie outside the sphere of radius equal to the chosen aperture, and that we call "interlopers". Columns 8 and 9 list the average ratios of the estimated-to-true velocity dispersion $\sqrt{3} \sigma_{a, p} / \sigma_{a}$, and of the estimated-to-true harmonic mean radius $\frac{\pi}{2} r_{a, p} / r_{a}$, respectively, all quantities being estimated within the given aperture $a$ (note that the projected quantities are multiplied by the deprojection factors). Column 10 lists the average ratio of the estimated-totrue virial radius, $\tilde{r}_{\mathrm{v}} / r_{\mathrm{v}}$. Columns 11 and 12 list the average ratios of the two mass estimates (the virial estimate and the velocitydispersion based estimate, respectively) to the true mass, $\tilde{M}_{\mathrm{v}} / M_{\mathrm{v}}$ and $M_{\sigma} / M_{\mathrm{v}}$, respectively. The dispersions of the quantities listed in Cols. 6-12 are given (within brackets) in the line immediately below. Columns 13 and 14 list the fractions of cluster projections for which the virial mass is a strong under- or over-estimate of the true mass, $\tilde{M}_{\mathrm{v}} / M_{\mathrm{v}}<1 / 2$, and $\tilde{M}_{\mathrm{v}} / M_{\mathrm{v}}>2$, respectively. 
Table 1. The ratios between the estimated dynamical quantities in projection and the true ones. Quantities in brackets are the dispersions on the estimated quantities.

\begin{tabular}{|c|c|c|c|c|c|c|c|c|c|c|c|c|c|}
\hline Id. & $\begin{array}{c}a \\
\left(h^{-1} \mathrm{Mpc}\right)\end{array}$ & $N$ & Tracer & Sel. & $N_{m}$ & $f_{\text {out }}$ & $\sqrt{3} \sigma_{a, p} / \sigma_{a}$ & $\frac{\pi}{2} r_{a, p} / r_{a}$ & $\tilde{r}_{\mathrm{v}} / r_{\mathrm{v}}$ & $\tilde{M}_{\mathrm{v}} / M_{\mathrm{v}}$ & $M_{\sigma} / M_{\mathrm{v}}$ & $f_{<0.5}$ & $f_{>2}$ \\
\hline 1 & 1.50 & 500 & $\overline{\mathrm{DM}}$ & $\overline{\mathrm{Rdm}}$ & $\begin{array}{l}402 \\
(48)\end{array}$ & $\begin{array}{c}0.18 \\
(0.11)\end{array}$ & $\begin{array}{c}0.93 \\
(0.10)\end{array}$ & $\begin{array}{c}1.22 \\
(0.12)\end{array}$ & $\begin{array}{c}1.08 \\
(0.10)\end{array}$ & $\begin{array}{c}1.10 \\
(0.31)\end{array}$ & $\begin{array}{c}0.88 \\
(0.28)\end{array}$ & 0.01 & 0.03 \\
\hline 2 & projection & with & abclusters exc & uded & $\begin{array}{l}396 \\
(50)\end{array}$ & $\begin{array}{c}0.17 \\
(0.10)\end{array}$ & $\begin{array}{c}0.90 \\
(0.10)\end{array}$ & $\begin{array}{l}1.21 \\
(0.11)\end{array}$ & $\begin{array}{c}1.06 \\
(0.08)\end{array}$ & $\begin{array}{l}1.02 \\
(0.25)\end{array}$ & $\begin{array}{c}0.81 \\
(0.24)\end{array}$ & 0.00 & 0.00 \\
\hline 3 & 1.50 & 100 & $\mathrm{DM}$ & $\mathrm{Rdm}$ & $\begin{array}{l}80 \\
(9)\end{array}$ & $\begin{array}{c}0.18 \\
(0.11)\end{array}$ & $\begin{array}{c}0.93 \\
(0.14)\end{array}$ & $\begin{array}{c}1.20 \\
(0.19)\end{array}$ & $\begin{array}{c}1.07 \\
(0.14)\end{array}$ & $\begin{array}{l}1.10 \\
(0.46)\end{array}$ & $\begin{array}{c}0.81 \\
(0.38)\end{array}$ & 0.04 & 0.10 \\
\hline 4 & 1.50 & 20 & $\mathrm{DM}$ & $\mathrm{Rdm}$ & $\begin{array}{l}17 \\
(2)\end{array}$ & $\begin{array}{c}0.20 \\
(0.14)\end{array}$ & $\begin{array}{c}0.89 \\
(0.27)\end{array}$ & $\begin{array}{c}1.37 \\
(0.28)\end{array}$ & $\begin{array}{c}1.11 \\
(0.25)\end{array}$ & $\begin{array}{l}1.25 \\
(0.79)\end{array}$ & $\begin{array}{c}0.83 \\
(0.58)\end{array}$ & 0.09 & 0.25 \\
\hline 5 & 1.50 & 500 & $\mathrm{DM}$ & Slit & $\begin{array}{l}198 \\
(51)\end{array}$ & $\begin{array}{c}0.26 \\
(0.13)\end{array}$ & $\begin{array}{c}0.90 \\
(0.12)\end{array}$ & $\begin{array}{l}1.63 \\
(0.17)\end{array}$ & $\begin{array}{l}1.18 \\
(0.10)\end{array}$ & $\begin{array}{l}1.51 \\
(0.41)\end{array}$ & $\begin{array}{c}0.83 \\
(0.28)\end{array}$ & 0.00 & 0.16 \\
\hline 6 & 0.50 & 500 & $\mathrm{DM}$ & $\mathrm{Rdm}$ & $\begin{array}{l}459 \\
(46)\end{array}$ & $\begin{array}{c}0.28 \\
(0.09)\end{array}$ & $\begin{array}{c}0.94 \\
(0.10)\end{array}$ & $\begin{array}{c}1.20 \\
(0.07)\end{array}$ & $\begin{array}{c}0.97 \\
(0.09)\end{array}$ & $\begin{array}{l}1.08 \\
(0.32)\end{array}$ & $\begin{array}{c}0.87 \\
(0.29)\end{array}$ & 0.01 & 0.02 \\
\hline 7 & 1.50 & 39 & G & All & $\begin{array}{r}30 \\
(12)\end{array}$ & $\begin{array}{c}0.26 \\
(0.15)\end{array}$ & $\begin{array}{c}0.95 \\
(0.27)\end{array}$ & $\begin{array}{c}1.39 \\
(0.25)\end{array}$ & $\begin{array}{c}1.16 \\
(0.24)\end{array}$ & $\begin{array}{c}1.40 \\
(0.85)\end{array}$ & $\begin{array}{c}0.94 \\
(0.62)\end{array}$ & 0.11 & 0.27 \\
\hline 8 & 1.50 & 39 & $\mathrm{DM}$ & $\mathrm{Rdm}$ & $\begin{array}{r}29 \\
(12)\end{array}$ & $\begin{array}{c}0.24 \\
(0.14)\end{array}$ & $\begin{array}{c}0.98 \\
(0.26)\end{array}$ & $\begin{array}{c}1.37 \\
(0.26)\end{array}$ & $\begin{array}{c}1.17 \\
(0.24)\end{array}$ & $\begin{array}{l}1.46 \\
(0.89)\end{array}$ & $\begin{array}{c}1.03 \\
(0.68)\end{array}$ & 0.03 & 0.31 \\
\hline 9 & 1.50 & 19 & $\mathrm{G} z_{\mathrm{f}} \geq 1.25$ & All & $\begin{array}{l}16 \\
(8)\end{array}$ & $\begin{array}{c}0.18 \\
(0.15)\end{array}$ & $\begin{array}{c}0.89 \\
(0.30)\end{array}$ & $\begin{array}{c}1.21 \\
(0.30)\end{array}$ & $\begin{array}{c}1.04 \\
(0.26)\end{array}$ & $\begin{array}{l}1.01 \\
(0.74)\end{array}$ & $\begin{array}{c}0.80 \\
(0.64)\end{array}$ & 0.20 & 0.15 \\
\hline 10 & 1.50 & 19 & $\mathrm{DM}$ & $\mathrm{Rdm}$ & $\begin{array}{l}15 \\
(7)\end{array}$ & $\begin{array}{c}0.25 \\
(0.18)\end{array}$ & $\begin{array}{c}0.93 \\
(0.37)\end{array}$ & $\begin{array}{c}1.41 \\
(0.32)\end{array}$ & $\begin{array}{c}1.13 \\
(0.35)\end{array}$ & $\begin{array}{c}1.32 \\
(1.14)\end{array}$ & $\begin{array}{c}0.92 \\
(0.83)\end{array}$ & 0.14 & 0.34 \\
\hline
\end{tabular}

\subsection{Projection effects}

In order to quantify the importance of projection effects on cluster mass estimates, we first consider a large number (500) of reliable tracers of the gravitational potential (DM particles), with no errors on their velocities, positions, and no radial incompleteness bias (see Table 1, Id. No. 1). The 500 DM particles are randomly selected along each of three orthogonal line-of-sights for each cluster, within a cylinder of $1.5 h^{-1} \mathrm{Mpc}$ radius. Interlopers are then rejected using the procedure described in Sect. 3. The case studied here is of course an idealized situation never achieved observationally, but this analysis serves to emphasize the importance of projection effects on cluster mass estimates, independently of any other observational bias.

On average, both the virial mass estimate and the $M_{\sigma}$ estimate are accurate to $\simeq 10 \%$, but the scatter around the true mass values for the different projections is quite large (see Fig. 6). The virial estimate somewhat overestimates the true mass, and this is caused by an overestimate of the harmonic mean radius, while $M_{\sigma}$ somewhat underestimates the true mass, and this is caused by an underestimate of the velocity dispersion (see Table 1).

In order to better understand the effects of projection, we examine the projected phase-space distribution of a stacked cluster in some detail. The stacked cluster is built as follows. In each cluster of the simulation set we randomly select 500 DM particles along three orthogonal projections, and identify the cluster members. We then normalize the clustercentric distances of these particles by the virial radius of the cluster they belong to, and their velocities (relative to their cluster mean velocity) by the velocity dispersion of the cluster they belong to, computed within one virial radius. We then stack the 62 clusters using the normalized radii and velocities. Finally, we return to physical units by multiplying the normalized radii and velocities of the DM particles in the stacked cluster, respectively by the average virial radius $\left(0.99 h^{-1} \mathrm{Mpc}\right)$, and by the average velocity dispersion $\left(657 \mathrm{~km} \mathrm{~s}^{-1}\right)$ of the 62 clusters.

In Fig. 7 we show the projected phase-space distribution of a randomly selected subset of the DM particles in the stacked

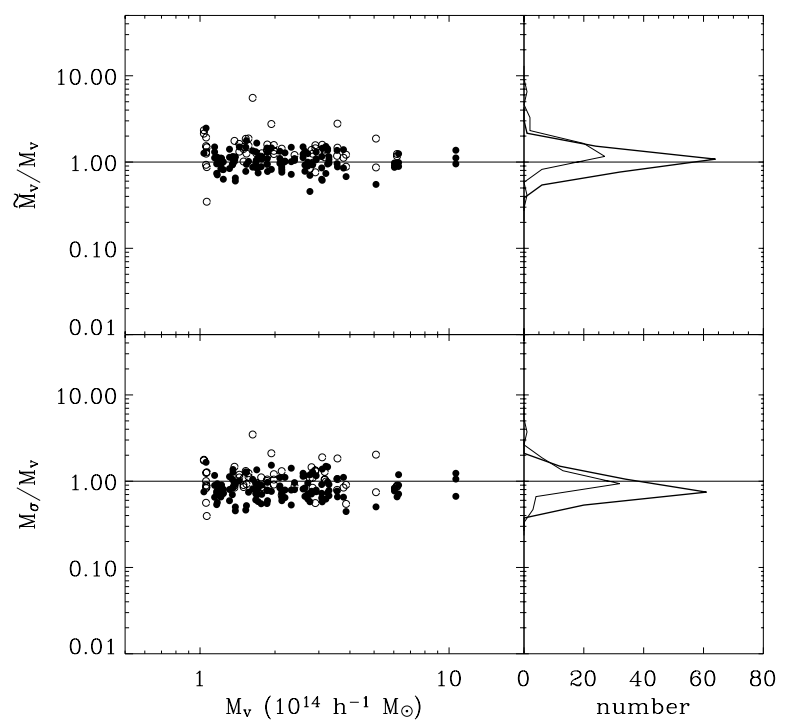

Fig. 6. The comparison between the masses estimated using only projected phase-space information and the true cluster masses. Top-left panel: the ratio between the virial and the true mass, $\tilde{M}_{\mathrm{v}} / M_{\mathrm{v}}$ vs. the true mass $M_{\mathrm{v}}$. Filled dots identify those projections where no significant evidence for substructure is found at the $0.01 \mathrm{c} .1$. Top-right panel: distributions of $\tilde{M}_{\mathrm{v}} / M_{\mathrm{v}}$ for the projections with and without significant evidence for substructure (thin and thick line, respectively). Bottom panels: same as top panels, but for $M_{\sigma}$ in lieu of $\tilde{M}_{\mathrm{v}}$. The masses $\tilde{M}_{\mathrm{v}}$ and $M_{\sigma}$ are estimated from projected distributions of DM particles. Initially, $500 \mathrm{DM}$ particles are randomly selected along three orthogonal line-of-sights in each cluster, within a cylinder of $1.5 h^{-1} \mathrm{Mpc}$ radius. Interlopers are then rejected using the procedure described in Sect. 3. Note that the vertical range is different from that of Fig. 5 and the vertical axis is now logarithmic.

cluster. Three orthogonal projections are stacked together in the figure. Different symbols are used to represent: i) DM particles contained within a sphere of $1.5 h^{-1} \mathrm{Mpc}$ radius and selected as cluster members by the interloper rejection procedure (filled squares); ii) DM particles located within this same sphere yet 


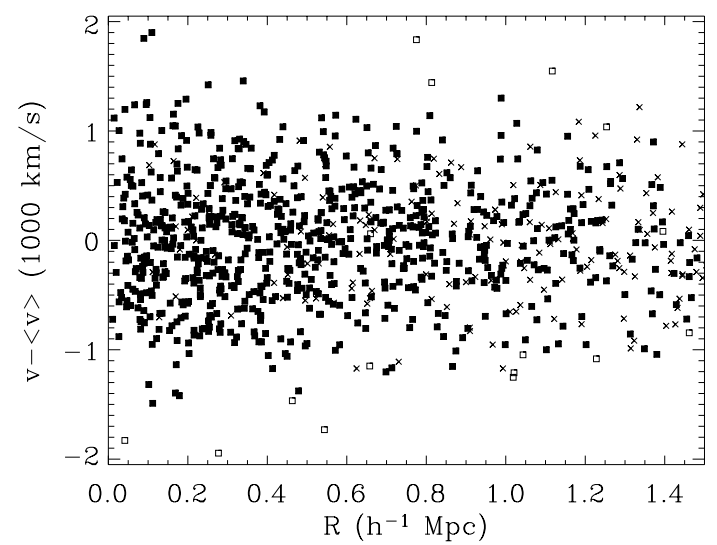

Fig. 7. Line-of-sight velocity vs. projected clustercentric distance for a subset of the DM particles in the stacked cluster (see text). Filled squares indicate DM particles contained within a sphere of $1.5 h^{-1} \mathrm{Mpc}$ radius and selected as cluster members by the interloper rejection procedure, open squares indicate DM particles located within this same sphere yet incorrectly rejected as interlopers, and $\mathrm{x}$ symbols indicate DM particles located outside this same sphere and yet selected as cluster members (interlopers).

incorrectly rejected as interlopers (open squares); and iii) DM particles located outside this same sphere and yet selected as cluster members ( $\times$ symbols). Clearly, the interloper rejection technique excludes only a few DM particles that are in fact within the $1.5 h^{-1} \mathrm{Mpc}$ sphere, but keeps several DM particles that are outside the $1.5 h^{-1} \mathrm{Mpc}$ sphere but have projected velocities that make them indistinguishable from the real cluster members in the projected phase-space diagram (what we have called interlopers, see Sect. 4).

The underestimate of the velocity dispersion of the cluster is partly due to the rejection of real cluster members at relatively high velocities. Most of the underestimate is however due to the inclusion of interlopers that are currently infalling toward the cluster along a filament. These interlopers lie within $\lesssim 10 h^{-1} \mathrm{Mpc}$ from the cluster centre (more distant DM particles have quite a different recession velocity from the cluster mean and are rejected by the interloper technique) and their infall motion makes their velocities resemble the average cluster velocity. In addition, they have an intrinsically small velocity dispersion within their filament. As a consequence, the projected velocity dispersion of these interlopers turns out to be smaller than that of the cluster within the selected aperture. On the other hand, their spatial distribution is not as centrally concentrated as that of the cluster members within the selected aperture, as shown in Fig. 8. This causes the harmonic mean radius to be overestimated.

Therefore, interlopers cause the overestimate of the harmonic mean radius, and, at the same time, the underestimate of the velocity dispersion, as already noted by C97 and Diaferio et al. (1999). Since the strongest of the two effects is the harmonic mean radius overestimate, also the virial mass is overestimated. On the other hand, $M_{\sigma}$ provides an underestimate of the mass because it only depends on the velocity dispersion estimate.

Despite these problems, the interloper selection technique seems to work reasonably well. On average only $\sim 1 \%$ of the real cluster members are rejected as interlopers, while on average $18 \%$ of the selected members are unrecognized interlopers (see $f_{\text {out }}$ in Table 1). However, projection effects can become critical for individual cases. In $3 \%$ of the cases the cluster masses are overestimated by a factor $>2$, and in $1 \%$ of the cases they

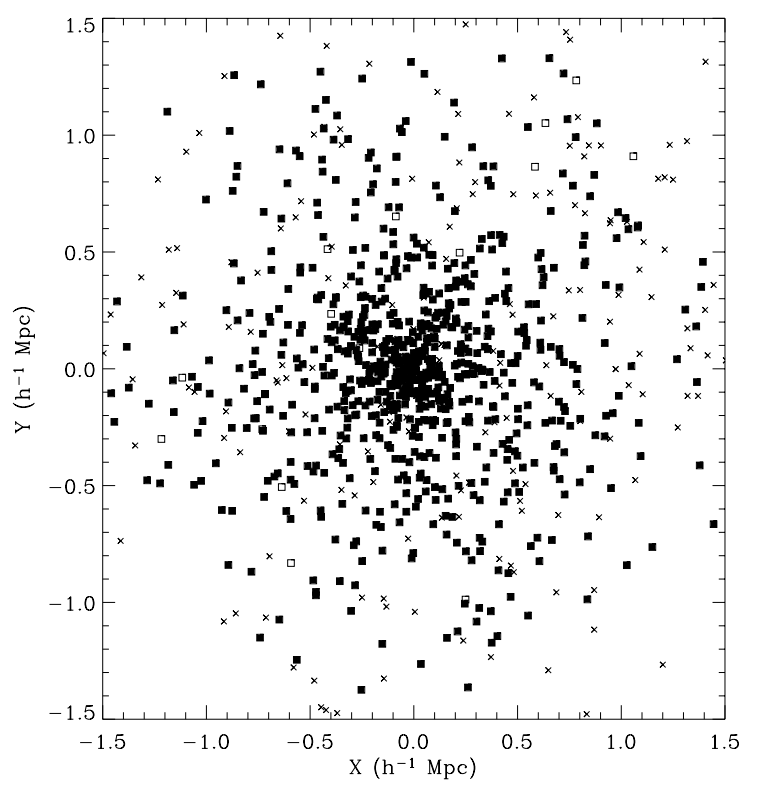

Fig. 8. The projected spatial distribution of the same DM particles shown in Fig. 7. Symbols are the same as in Fig. 7.

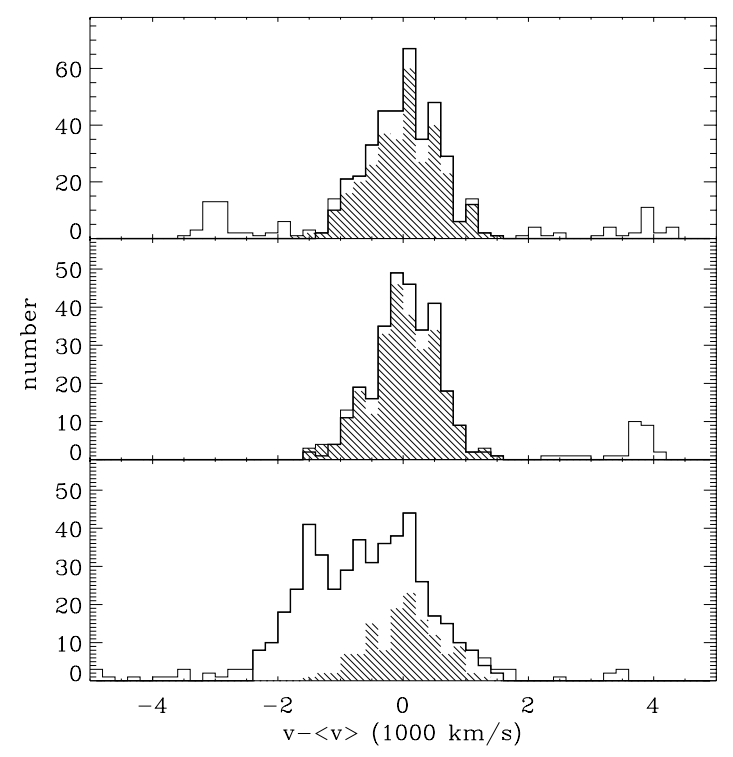

Fig. 9. The velocity distributions of a random subset of DM particles of a simulated cluster, along three orthogonal projections. Thin lines indicate the velocity distributions of all DM particles within a cylinder of radius $1.5 h^{-1} \mathrm{Mpc}$, thick lines the velocity distributions of those DM particles identified as cluster members, and the hatched histograms show the velocity distributions of the DM particles located within a sphere of $1.5 h^{-1} \mathrm{Mpc}$ radius.

are underestimated by a factor $<1 / 2$, using the virial mass estimator (see Fig. 6, and the last two columns in Table 1). Large errors in the virial mass estimates correspond to extreme cases of failure of the interloper rejection algorithm, when either a compact dynamical system is artificially split in two by the weighted gap algorithm, or, more frequently, a physically distinct group is merged together with the main cluster. This last case is illustrated in Fig. 9, where we plot the velocity distributions of i) the DM particles within a sphere of $1.5 h^{-1}$ Mpc radius (hatched histogram); ii) all DM particles within the cylinder of same radius (thin line); and iii) the DM particles identified as cluster members (thick line). In one of the three projections (bottom panel of 
Fig. 9) the cluster velocity distribution is heavily contaminated by that of a foreground group, and the interloper rejection technique fails to isolate the cluster members. Note, however, that large errors in the mass estimates generally occur only for one of the three orthogonal projections considered per cluster.

\subsection{Subclustering}

The presence of subclustering has long been thought to potentially cause incorrect cluster mass estimates (e.g. Bird 1995). Several algorithms have been developed over the years to address this problem (see, e.g., Girardi \& Biviano 2002, and references therein). One of the most commonly used (and easy to implement) algorithms is that of Dressler \& Shectman (1988, DS hereafter). Here we apply this algorithm (or, actually, Biviano et al.'s 2002, version of it) to the simulated clusters seen along each of three orthogonal projections. As before (see Sect. 4.1) $500 \mathrm{DM}$ particles are randomly selected within a cylinder of $1.5 h^{-1} \mathrm{Mpc}$ radius; the DS test is applied on those particles that are selected as cluster members.

In 59 cases out of 186 the DS test provides a probability $\geq 0.99$ that the cluster, as seen along the chosen projection, has significant subclustering. The cluster projections with $\geq 0.99$ subclustering probability are also those with the largest mass overestimates. As an example, in the case-study illustrated in Fig. 9, subclustering is detected with high significance along the projection shown in the bottom panel. Among the 127 cluster projections without evidence for subclustering, only one has its virial mass overestimated by a factor $>2$. As a consequence, the virial mass estimates are now closer to the true cluster masses, on average (see Fig. 6 and Table 1, Id. No. 2).

Hence the simple DS test appears to be a useful tool to identify those cases of large virial mass overestimates. However, the situation is not so simple. When only $100 \mathrm{DM}$ particles are selected instead of 500 , the DS test gives a probability $\geq 0.99$ only for 28 out of the 186 cluster projections. Clearly, when the sample size is reduced the DS statistics is less significant. Yet, observational results based on even smaller sample sizes, suggest a higher fraction of clusters with subclustering, $\sim 1 / 3$ (e.g. DS; Biviano et al. 1997). Hence the simulated clusters seem to display a lower amount of subclustering, on average, than real clusters.

\subsection{Undersampling}

In order to study how the accuracy of mass estimates depends on the number of cluster members available, we randomly select from 10 to $500 \mathrm{DM}$ particles within cylinders of $1.5 h^{-1} \mathrm{Mpc}$ radius, along three orthogonal projections. Cluster members are then selected using the interloper rejection procedure described in Sect. 3.

Reducing the sample size increases the scatter of the dynamical estimates and affects the virial mass estimates (see Fig. 10 and Table 1, Id. Nos. 1, 3, and 4). The average $r_{a, p} / r_{a}$ ratio tends to increase with decreasing number of selected cluster members, $N_{m}$, for $N_{m} \lesssim 60$, until it reaches a plateau for $N_{m} \lesssim 20$. On the other hand, the average $\sqrt{3} \sigma_{a, p} / \sigma_{a}$ ratio hardly changes with the size of the sample. As a consequence, for samples of $\$ 60$ cluster members, the simpler $M_{\sigma}$ estimate appears to be a better predictor of the true mass than the virial mass estimate, $\tilde{M}_{\mathrm{v}}$, which is affected by the bias of $r_{a, p}$.

The increasing overestimate of the harmonic mean radius (and hence of the virial mass) as the size of the sample decreases

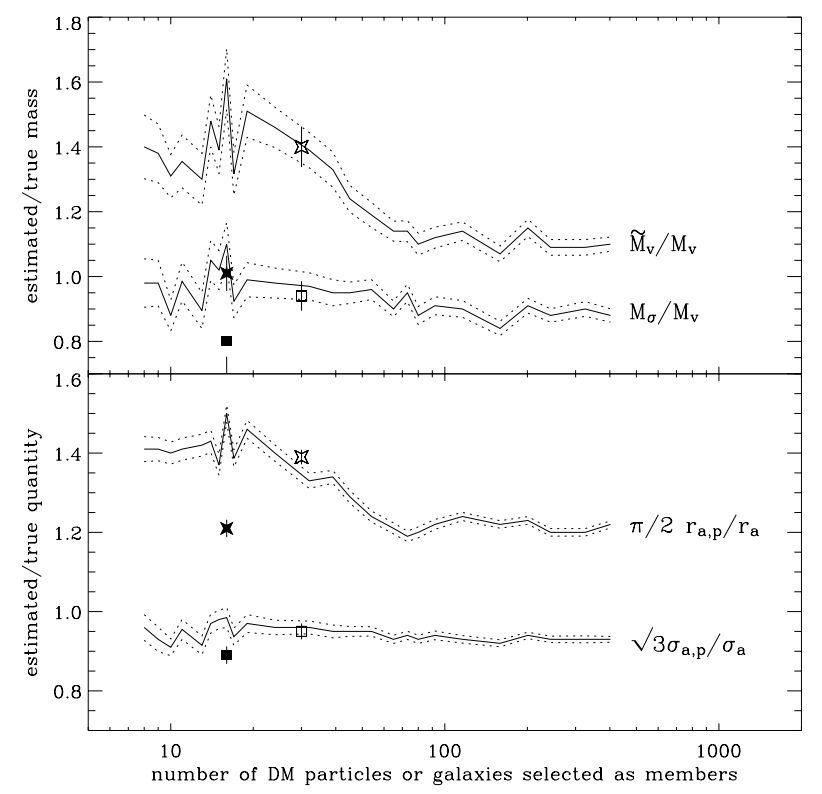

Fig. 10. The ratio of estimated to true dynamical quantities, averaged over all cluster projections, as a function of the number of DM particles selected as cluster members. Upper panel: $\tilde{M}_{\mathrm{v}} / M_{\mathrm{v}}$ (lines above) and $M_{\sigma} / M_{\mathrm{v}}$ (lines below). Lower panel: $\frac{\pi}{2} r_{a, p} / r_{a}$ (lines above) and $\sqrt{3} \sigma_{a, p} / \sigma_{a}$ (lines below). Dotted lines represent 1- $\sigma$ c.l. on the averages. The symbols represent the values obtained using galaxies (rather than DM particles) as tracers. Empty symbols are for all galaxies; filled symbols for galaxies with $z_{\mathrm{f}} \geq 1.25$ only. Stars refer to $\tilde{M}_{\mathrm{v}} / M_{\mathrm{v}}$ and $\frac{\pi}{2} R_{h} / r_{h}$, squares to $M_{\sigma} / M_{\mathrm{v}}$ and $\sigma_{200} / \sigma_{\mathrm{v}}$.

is mostly due to an increasing fraction of interlopers, from $18 \%$ for $N_{m} \gtrsim 60$, to $23-25 \%$ for $N_{m} \lesssim 40$. Part of the effect is however induced by a statistical bias that affects the harmonic mean radius estimate for small sample sizes. This can be seen by computing the harmonic mean radius in projection, but using only real cluster members. We find that in this case, the ratio $r_{a, p} / r_{a}$ remains close to unity for $N_{m} \gtrsim 60$, then starts increasing with decreasing $N_{m}$, until it becomes $\simeq 1.1$ for $N_{m} \simeq 20$.

\subsection{Radial-dependent incompleteness}

When a cluster field is observed in spectroscopy, completeness to a given limiting magnitude is rarely achieved. However, apart from the very bright cluster galaxies, there is no evidence for luminosity segregation in galaxy clusters (e.g. Biviano et al. 1992, 2002; Adami et al. 1998a; Cappi et al. 2003; Goto 2005; Yang et al. 2005). Hence a random selection of the available galaxies in a cluster field, even if not complete down to a given magnitude, should produce a sample with an unbiased projected phasespace distribution. In the previous analyses (Sects. 4.1-4.3) such a random selection was indeed applied. However, this is not always an easy task to accomplish observationally. In fact, the number of slits (fibers) per mask in multi-slit (multi-fiber) spectrographs is fixed, hence the central, high-density regions of galaxy clusters are often sampled to a brighter magnitude than the external regions. As a consequence, the spatial distribution of the galaxies selected for spectroscopy turns out to be more extended than the parent spatial distribution of cluster galaxies. Said otherwise, a higher fraction of galaxies is selected from the total in the outer regions, i.e. the incompleteness is not random but depends on radius. 
Ignoring the problem of radial-dependent incompleteness can have quite a catastrophic effect on the cluster mass estimate, as we show in the following. We simulate an observational set-up in which our clusters are observed with a multi-slit spectrograph with four quadrants, each 13 arcmin on a side, with a typical slit separation of $10 \operatorname{arcsec}$ (we remind the reader that the clusters have been set at a distance of $250 h^{-1} \mathrm{Mpc}$ from the observer). The results are listed in Table 1, Id. No. 5. In comparing these results to those obtained in the case of a random selection of tracers (Table 1, Id. No. 1), we note three differences. First, the average number of selected DM particles is only 198 out of the initial 500 randomly chosen in a cylinder of $1.5 h^{-1} \mathrm{Mpc}$ radius. Second, the fraction of interlopers is increased (26\% vs. $18 \%$ ). Third, the harmonic mean radius is strongly overestimated in projection. All these differences are due to the geometrical constraints imposed by the multi-slit single-mask observation, that forces a sparser sampling of the dense cluster core relatively to the cluster outskirts. The observational spatial distribution of the selected tracers is thus less centrally concentrated than the underlying parent distribution.

In order to avoid this bias, the central cluster regions must be observed with more masks per unit area than the external cluster regions. Alternatively, photometric observations can be used to estimate, and correct for, the radial incompleteness of the spectroscopic sampling. When neither option is viable, it is better to rely on the $M_{\sigma}$ estimate, which is much less affected by problems of incompleteness since cluster velocity dispersion profiles have only a mild radial dependence (see, e.g., Girardi et al. 1996 and Fig. 4).

\subsection{Other observational effects}

Other observational effects that could, in principle, affect a cluster mass determination, are i) a different size of the observational aperture (the radius of the cylinder within which the tracers are selected); ii) the uncertainty on the determination of the cluster centre; and iii) errors on the redshifts of cluster galaxies. All these effects can change the projected phase-space distributions of the tracers, thereby affecting in principle also the identification of cluster members.

In order to estimate the effect that different aperture sizes have on the cluster mass estimates, we compare the results obtained by selecting DM particles within cylinders of $1.5,0.75$, and $0.5 h^{-1} \mathrm{Mpc}$ radii. As the aperture $a$ of the cylinder is decreased, more particles are selected as cluster members out of the initial 500 distributed in the cylinder, but a higher fraction of the selected members are actually located outside a sphere of radius $a$. As a consequence, the results of the dynamical analysis are similar for different apertures (compare the results for an aperture of $0.5 h^{-1} \mathrm{Mpc}$ with those for an aperture of $1.5 h^{-1} \mathrm{Mpc}$, Id. Nos. 6 and 1, respectively, in Table 1).

In order to simulate the observational uncertainty in the determination of a cluster centre, a random offset is added to the position of the real cluster centre (see Sect. 3). The offset is randomly taken from a lognormal distribution with average of $40 \mathrm{kpc}$ and dispersion of $50 \mathrm{kpc}$, modeled after the observed distribution of uncertainties in the centre positions of ENACS clusters (Adami et al. 1998b). We find that centering errors of this size have essentially no effect on the accuracy of mass estimation.

Velocity errors contribute to increase the estimate of the cluster velocity dispersion. In order to simulate the observational errors on galaxy redshifts, a random velocity offset is added to the real DM particle velocity. The offset is randomly taken from

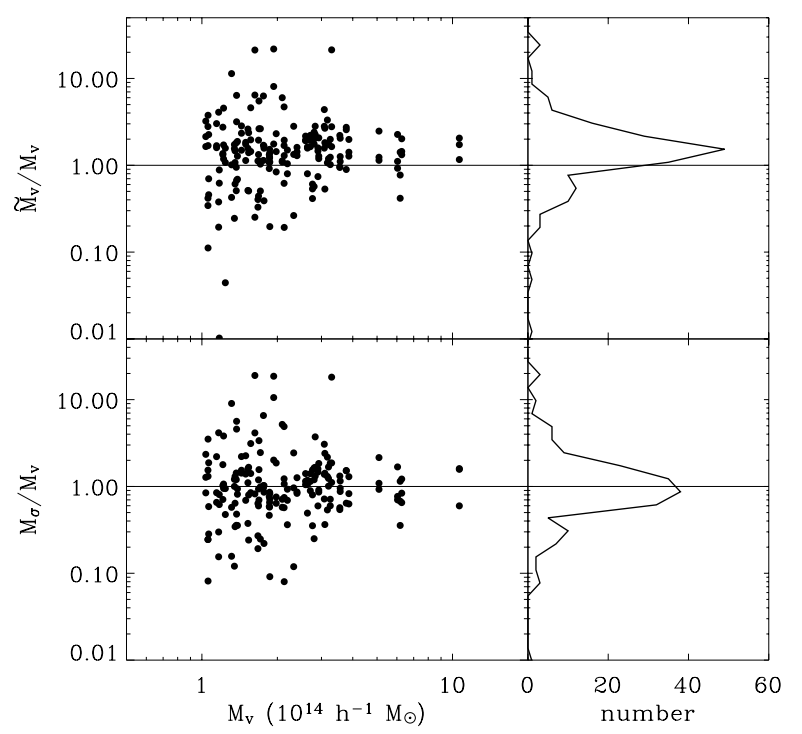

Fig. 11. Same as Fig. 6 but for galaxies instead of DM particles. No distinction is made here between projections with and without detection of substructures.

a Gaussian distribution with width of 70 or $210 \mathrm{~km} \mathrm{~s}^{-1}$. An error of $70 \mathrm{~km} \mathrm{~s}^{-1}$ is typical for observations of nearby clusters observations (see, e.g., Katgert et al. 1996), while an error three times larger corresponds to the observational uncertainties of galaxy redshifts in distant clusters (e.g. Demarco et al. 2005). As long as the velocity dispersion estimate is corrected following the prescriptions of Danese et al. (1980), the dynamical estimates are not significantly affected by errors on the velocities of the tracer particles. Note however that the 62 clusters used in our analysis have a median line-of-sight velocity dispersion of $622 \mathrm{~km} \mathrm{~s}^{-1}$. Galaxy velocity errors are likely to become more important for mass estimates of low-velocity dispersion groups.

\subsection{Galaxies}

The limited number of simulated galaxies per cluster makes it impossible to study in detail all kinds of different observational effects, as it was done for the DM particles. But galaxies have the advantage that early- and late-types can be distinguished, based on their formation redshift $z_{\mathrm{f}}$ (see Sect. 2). We start by considering all available galaxies within a cylinder of $1.5 h^{-1} \mathrm{Mpc}$ radius. The results are shown in Table 1, Id. No. 7 and Fig. 11. Dynamical estimates based on galaxies are significantly affected by the presence of interlopers. About $1 / 4$ of the selected cluster members are in fact outside the sphere of $1.5 h^{-1} \mathrm{Mpc}$ radius. As a consequence, both $r_{a, p}$ and $\tilde{M}_{\mathrm{v}}$ overestimate the harmonic mean radius and the mass, respectively, by about $40 \%$. In about $1 / 3$ of the projections, the virial mass estimates are wrong by a factor of two or greater. On the other hand, the $\sigma_{a, p}$ and $M_{\sigma}$ estimates are (on average) almost correct (to within 5-6\%).

In order to compare these results with those obtained using DM particles as tracers of the potential, we randomly select within each cylinder of $1.5 h^{-1} \mathrm{Mpc}$ radius a number of DM particles identical to that of the galaxies in that same cylinder. In this way we make sure that the effects of undersampling (see Sect. 4.3) are the same for the samples of galaxies and DM particles. The results are given in Table 1, Id. No. 8. The differences are very marginal.

When only early-type galaxies (i.e. those with $z_{\mathrm{f}} \geq 1.25$ ) are selected, the results of the dynamical analysis are quite 


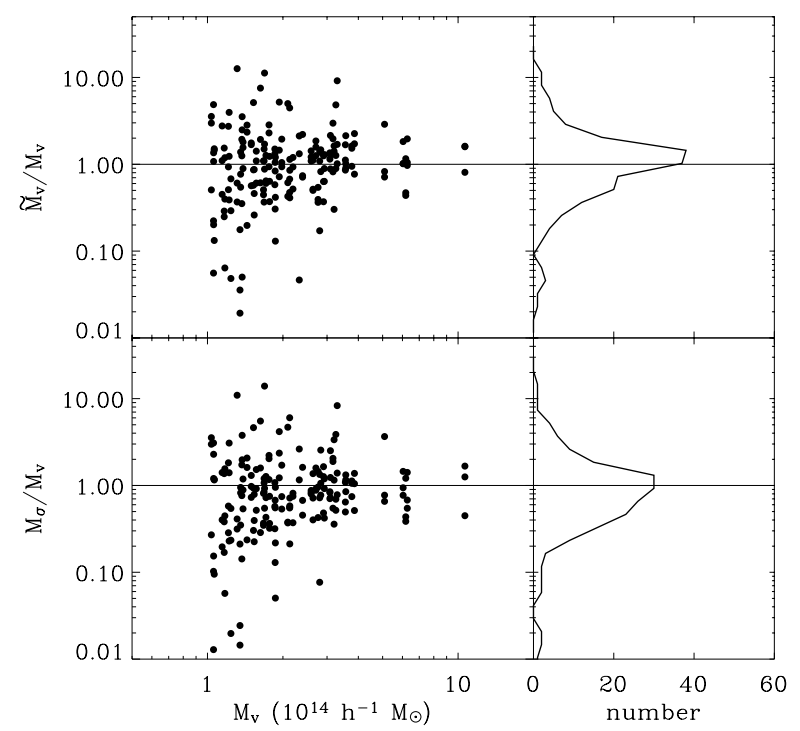

Fig. 12. Same as Fig. 11 but only for galaxies with $z_{\mathrm{f}} \geq 1.25$.

different from the case in which all galaxies are selected (compare Id. Nos. 9 and 7 in Table 1 respectively; see also Fig. 12). In particular, the average fraction of interlopers is now reduced to $18 \%$, and the average value of $\tilde{M}_{\mathrm{v}}$ is now almost identical to the real mass value. On the other hand, the $M_{\sigma}$ estimate is $20 \%$ too low, as a consequence of a dynamical segregation effect (see the discussion below). The number of clusters with a mass estimate wrong by a factor of at least two is still high, about $1 / 3$ of the total, but this is expected because of the large scatter in the mass estimates due to the small number of available tracers.

As before, we compare these results with those obtained by selecting the same number of DM particles as early-type galaxies, within the cylinder of $1.5 h^{-1} \mathrm{Mpc}$ radius. The results are listed in Table 1, Id. No. 10, and are quite different from those obtained using early-type galaxies, since, in particular, the fraction of interlopers is higher, and $\tilde{M}_{\mathrm{v}}$ significantly overestimates the true mass.

In order to understand the differences found between the mass estimates obtained using DM particles, all galaxies, and early-type galaxies, we consider their relative distributions in full phase-space. For this we build a stacked cluster, as in Sect. 2. The $3 \mathrm{~d}$ number density profiles of DM particles and galaxies in the stacked cluster are shown in Fig. 13. Galaxies are clearly less centrally concentrated than DM particles. This is confirmed by a Rank-Sum (RS hereafter) statistical test that reject the null hypothesis that the DM particles and the galaxies have the same radial distribution with a high confidence level (c.l. in the following), >0.999 (see also Nagai \& Kravtsov 2005). Interestingly, this is in agreement with the observations that show a decreasing mass-to-light ratio as a function of clustercentric distance (e.g. Biviano \& Girardi 2003; Rines et al. 2004). On the other hand, the distribution of the normalised velocities, $|v| / \sigma_{\mathrm{v}}$, of galaxies is not significantly different from that of DM particles (RS test c.l. 0.829; see Fig. 14).

The spatial distribution of early-type galaxies is significantly more concentrated than that of all galaxies (RS-test c.1. >0.999), and marginally more concentrated than that of DM particles (RS-test c.1. 0.939; see Fig. 13). The velocity distribution of early-type galaxies is significantly different from those of all the galaxies and the DM particles (RS-test c.l. >0.999; see Fig. 14). The high significance of this difference is a consequence of the large size of the data-sets, and does not reflect a large

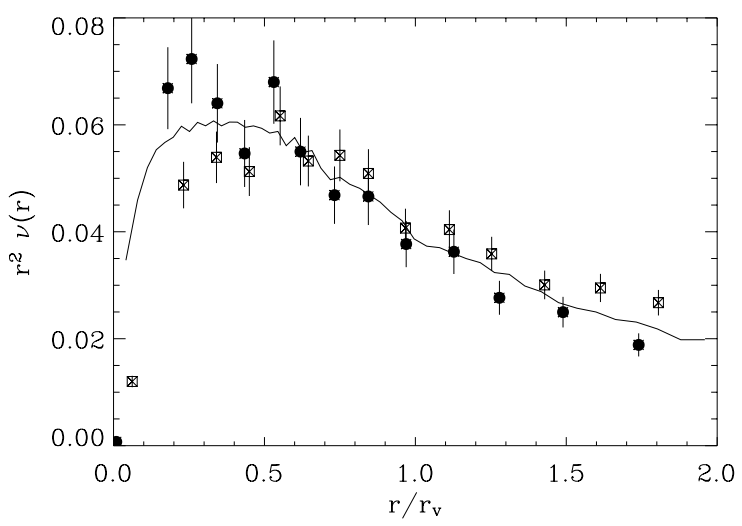

Fig. 13. The 3d number density profiles of DM particles (solid line), galaxies with $z_{\mathrm{f}} \geq 1.25$ (dots), and all galaxies (squared $\times$ 's) in the stacked cluster.

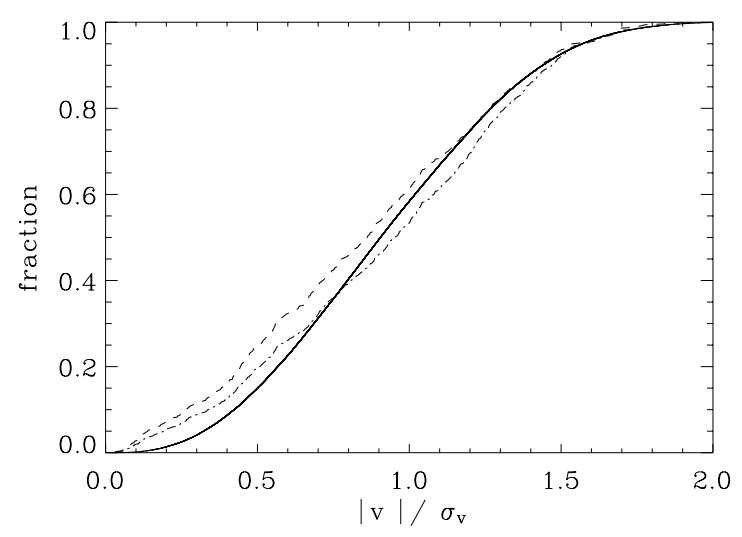

Fig. 14. The cumulative distributions of the 3-d velocities of DM particles (solid line), galaxies with $z_{\mathrm{f}} \geq 1.25$ (dashed line), and all galaxies (dash-dotted line) in the stacked cluster.

velocity bias: on average the modulus of the velocities of earlytype galaxies is 0.94 that of DM particles and 0.93 that of all the galaxies. In real clusters, the different velocity distribution of early- and late-type galaxies is a well established observational fact (Moss \& Dickens 1977; Sodré et al. 1989). The factor by which the velocity dispersion of early-type galaxies is lower than the velocity dispersion of all the cluster galaxies is 0.93 , on average, for the ENACS clusters (as can be derived from the estimates given in Biviano et al. 1997), a value identical to the one found here for simulated galaxies.

The different spatial distributions of all galaxies and DM particles does not seem to influence the results of the dynamical analysis in projected phase-space. One would expect the wider spatial distribution of galaxies to result in a larger $r_{a}$, and hence $r_{a, p}$ estimate. However, the $r_{a, p}$ estimate is dominated by the presence of interlopers. Since the fractions of interlopers among galaxies and among DM particles are similar, also the $r_{a, p}$ estimates are similar.

When only early-type galaxies are selected, the fraction of interlopers decreases because the fraction of early-type galaxies outside clusters is low, $29 \%$, much lower than the fraction of early-type galaxies within clusters (see Sect. 2). As a consequence, also the cluster $r_{a, p}$ estimate is closer to the real $r_{a}$ value, than in the case of DM particles, although still $\simeq 20 \%$ too high. The $r_{a}$ overestimate does not result in an overestimate of the virial mass because $\sigma_{a, p}$ underestimates the real $\sigma_{a}$ by $\simeq 10 \%$. This underestimate is caused by the narrower velocity distribution of early-type galaxies relatively to DM particles. 
In conclusion, galaxies have a biased distribution relative to DM particles. Considering all the cluster galaxies, they have a wider spatial distribution than DM particles, but a similar velocity distribution. Considering instead only "early-type" galaxies, these have both a narrower spatial distribution and a narrower velocity distribution than DM particles. Differences in spatial distributions have no effect on the dynamical mass estimates, since the estimate of the harmonic mean radius is dominated by the influence of interlopers. Selecting early-type galaxies helps improve the accuracy of the virial mass estimate because fewer interlopers enter the sample. On the other hand, the different velocity distribution of early-type galaxies with respect to that of DM particles results in $M_{\sigma}$ estimates that are too small.

\section{Discussion}

We have used a sample of 62 galaxy clusters extracted from a cosmological simulation, each with at least 10 galaxies within the cluster virial radius, to study the reliability of cluster mass estimates based on the distribution of their member galaxies. Galaxies in the simulation have been typed "early" or "late" based on the average formation redshift of their stellar population. The projected phase-space distributions of both the earlyand the late-type galaxies of the simulation have been shown to be similar to those observed in real galaxy clusters (see Sect. 2).

Two mass estimators have been considered in our analysis. One is the classical virial theorem estimate (corrected for the surface pressure term), and the other is an estimate based entirely on the cluster velocity dispersion $\left(M_{\sigma}\right.$, see Sect. 3). By application to the set of simulated clusters in full phase-space, we have shown these estimators to be unbiased, and the clusters to be virialized (on average) as expected for $z \sim 0$ clusters in a $\Lambda$ CDM cosmology.

In order to study how efficient these mass estimators are when applied to observed clusters, we have analysed our set of 62 simulated clusters in projection. Three orthogonal projections have been considered for each cluster. DM particles or galaxies have been selected in cylinders of given aperture radius and $\simeq 200 h^{-1} \mathrm{Mpc}$ depth, in order to simulate the effect of interlopers. Cluster members have then been selected with methods commonly used in the recent literature (see Sect. 3). DM particles have been considered as tracers of the potential, instead of galaxies, when we wanted to explore the effects of particular observational conditions with sufficiently good statistics. However, the results of the dynamical analysis are very similar when using the same number of DM particles or galaxies, except when galaxies of a given type are specifically selected. Hence we think that our conclusions based on the analyses of clusters of DM particles should be applicable to the real world, when no distinction is made between galaxies of different types.

Projection effects significantly affect the reliability of cluster mass estimates through the inclusion of interlopers among the samples of presumed cluster members (see Sect. 4.1). They are responsible for large errors in the mass estimates of some clusters, depending on the projection direction. However, despite a few catastrophic cases, the virial mass estimates are, on average, within $10 \%$ of the true values, if a sufficient number of DM particles are used as tracers of the potential, $\gtrsim 60$. Nowadays, having a spectroscopic sample of $\gtrsim 60$ member galaxies per cluster does not represent a challenging observational target, even for distant clusters (Demarco et al. 2005), thanks to the multiplexing capabilities of instruments like VIMOS at the VLT (e.g. Czoske et al. 2002) and LRIS at Keck (e.g. Goto et al. 2005). This was not the case for large spectroscopic surveys of galaxy clusters in the past (Katgert et al. 1996; Carlberg et al. 1997b).

Subclustering and the presence of groups along the lineof-sight to a cluster are responsible for the few catastrophic cases of strong mass under- and overestimation. Using the simple DS-test for subclustering it is however possible to identify the worst cases and eventually remove them from the sample (see Sect. 4.2). Similar conclusions have already been reached by vH97 on their simulated clusters, and by Bird (1995) observationally. Note however that it becomes increasingly difficult to identify clusters whose masses are overestimated because of subclustering, when the size of the data-sample is decreased. Even with $\sim 100$ tracers of the gravitational potential per cluster, 3/4 of the cases of significant subclustering previously found using 500 tracers can no longer be identified.

The (positive) bias of virial mass estimates strongly increases as the number of tracers of the potential is decreased below $\sim 60$ (see Sect. 4.3). Since cluster velocity dispersions are always slightly underestimated (by $\sim 5 \%$ ) the bias has to do with an overestimate of the harmonic mean radius. This in turn has its origin in the presence of interlopers among the particles identified as members. As already noticed by C97, these interlopers are characterized by a kinematic component of their velocities that cancels the difference in the cosmological components of the particle and cluster velocities, i.e., these DM particles are infalling into the cluster. Since they are outside the cluster, their spatial distribution is not as centrally concentrated as that of real cluster member particles, hence they contribute to increase the harmonic mean radius estimate. Diaferio et al. (1999) came to essentially the same conclusions.

In order to reduce the mass discrepancies when $\$ 60$ members per clusters are available, one could take advantage of the fact that the $M_{\sigma}$ estimates become less biased as the size of the sample is decreased, i.e., if the sampling of a cluster is poor, it is better to use $M_{\sigma}$ rather than the virial mass. This is also the case when there is considerable uncertainty in the completeness of the selected sample of tracers (see Sect. 4.4). Sampling the same number of tracers within denser cluster regions (i.e. reducing the aperture size of the observational set-up) does not significantly improve the virial mass estimate, but could be observationally more convenient.

When using galaxies instead of DM particles as tracers, it is possible to draw subsamples selected on the basis of the galaxy properties. When only early-type galaxies are selected, the bias in the virial mass estimate is strongly suppressed, even if the average number of cluster members is very small. A similar conclusion was reached observationally by Biviano et al. (1997), who suggested to exclude emission-line galaxies from the sample of objects to be used in a cluster virial mass determination (see also Sanchis et al. 2004). The improvement in the virial mass estimate obtained using only early-type galaxies depends on the fact that the fraction of early-type galaxies is higher among cluster members than in the field, the so-called morphology-density relation (Dressler 1980) which is evident also in our simulated clusters. Hence, the interloper contamination is substantially reduced, compared to the case in which all galaxies (or DM particles) are selected.

A comparison of our results with previous works is not straightforward, because of several differences in the analyses. Some of the previous works did not use the virial mass estimates at the estimated virial radius, but only the $\sigma_{\mathrm{v}}$ estimate (Frenk et al. 1990; vH97), or isothermal mass estimates derived from $\sigma_{\mathrm{v}}$ (C97; RB99), without using the spatial 
distribution of cluster members (i.e. the harmonic mean radius estimate). The $M_{\sigma}$ estimate used here differs from the isothermal mass estimates used in C97 and RB99 because their masses are derived within fixed linear radii, and hence have a $\sigma_{\mathrm{v}}^{2}$ dependence, while $M_{\sigma}$ is the mass within $r_{\mathrm{v}}$, and hence has a $\sigma_{\mathrm{v}}^{3}$ dependence. Both Sanchis et al. (2004) and Łokas et al. (2005) derived the cluster masses from the application of the isotropic Jeans equation. Since this method requires a sufficient number of tracers, Sanchis et al. (2004) and Łokas et al. (2005) only considered the case where 400, respectively 300, DM particles are available in each simulated cluster.

Most previous analyses were based on the classical 3- $\sigma$ clipping method of Yahil \& Vidal (1977), while the algorithm for the identification of interlopers used in this work is more sophisticated. However, the methods used by Sanchis et al. (2004) and Łokas et al. (2005) have several similarities with the one used in this paper. Moreover, vH97 did consider the method of den Hartog \& Katgert (1996), which is part of the technique used in this paper (see Sect. 3); vH97 indeed concluded that this method performs better than Yahil \& Vidal's, particularly in the presence of substructures. We do not expect to see a radical change in the average performance of the virial mass estimator as a consequence of using a different interloper rejection technique. First of all, it has been shown by several authors that different interloper rejection techniques generally lead to similar cluster mass estimates, when the number of sampled galaxies is sufficiently large (e.g. Girardi et al. 1993; Adami et al. 1998c; Biviano \& Girardi 2003; Pimbblet et al. 2005). Since a cluster velocity dispersion decreases with radius (see Fig. 7; see also den Hartog \& Katgert 1996; Biviano \& Katgert 2004) an interloper rejection method based on galaxy velocities only (like, e.g., Yahil \& Vidal's) will probably reject more cluster members near the cluster centre, while accepting more interlopers at the cluster edge, relative to a method that takes into account galaxy velocities and positions. However, the radial gradient of a cluster velocity dispersion is not strong, and the effect of neglecting the radial variation of a cluster velocity dispersion on the estimate of global cluster quantities, like its mass and velocity dispersion, is likely to be negligible, unless there is substantial substructure. Substructure is definitely identified much more efficiently by using the combined spatial and velocity information than with velocity information alone (see, e.g., Girardi \& Biviano 2002, and references therein). A detailed comparison of various methods of interloper rejection is however deferred to a forthcoming paper (Girardi et al., in preparation).

Both Sanchis et al. (2004) and Łokas et al. (2005) concluded that the virial masses of clusters can be reliably estimated using several hundreds of tracers of the gravitational potential, and we substantially agree on this. The method they used to estimate cluster masses are however different from the methods we considered in this paper. Both Sanchis et al. (2004) and Łokas et al. (2005, see their Fig. 2) found that their method yields a mild systematic underestimate of the cluster masses. On the other hand, for similar numbers of tracers, our methods provide mass estimates within $\simeq \pm 10 \%$ of the true values. Their method is however aimed at deriving not only cluster masses, but also internal velocity anisotropies of the tracers population.

The rôle of interlopers in the overestimate of the harmonic mean radius was emphasized by C97 and Diaferio et al. (1999), and is in agreement with our findings. The systematic underestimate of $\sigma_{\mathrm{v}}$ by den Hartog \& Katgert's (1996) method was already noted by $\mathrm{vH} 97$, but the effect was claimed to be larger than we see here, $\sim 100 \mathrm{~km} \mathrm{~s}^{-1}$ vs. our estimate of only $\lesssim 50 \mathrm{~km} \mathrm{~s}^{-1}$ on average. $\mathrm{C} 97$ and $\mathrm{vH} 97$ suggested that the mass estimate bias decreases with the mass of the system, and increases with the aperture used to select the tracers. In our analysis we find that these trends are very marginal, if they are present at all. Perhaps this is due to our use of a more efficient interloper rejection algorithm.

The issue of a velocity bias of galaxies relatively to DM has been raised several times, with some authors claiming it to be rather strong (Frenk et al. 1996), and others rather mild (Berlind et al. 2003; Faltenbacher et al. 2005). We find that the result depends on which galaxies are selected, since the bias is negligible when all galaxies are considered, but significant, although small (0.94, on average), when only early-type galaxies are considered (see Sect. 4.6). The dependence of the velocity bias on galaxy mass will be explored in a future paper.

\section{Conclusions}

Using a set of 62 clusters extracted from a $\Lambda \mathrm{CDM}$ cosmological simulation, we investigated the behaviour of mass estimators based on the projected phase-space of galaxies. Several observational effects were considered, like the presence of interlopers and subclusters, the sample size, the size of the observational aperture, incompleteness and the selection of different tracers of the gravitational potential (early-type galaxies, all galaxies, DM particles).

Our results show that the virial mass estimator is almost unbiased for samples of $\gtrsim 60$ tracers. The average bias is 1.10 , with a scatter of $0.30(0.40)$ for samples of 400 (60) cluster members. For smaller data-sets, the bias of the virial mass estimator increases with decreasing sample sizes, reaching a maximum of $\simeq 1.5-1.6$ when the number of cluster members is decreased to $\simeq 15-20$. The virial mass estimates can be improved by removing clusters with significant evidence of subclustering, or by selecting early-type galaxies as tracers. The $M_{\sigma}$ estimator, based entirely on the cluster velocity dispersion, has a bias of $\simeq 0.90$ for sample sizes $\gtrsim 60$ members, and is essentially unbiased for smaller data-sets. The scatter of the $M_{\sigma}$ estimator increases from $\sim 0.30$ for samples of $\gtrsim 60$ cluster members, to $\sim 0.60$ for samples of only 10 cluster members. Since early-type galaxies have on average a lower velocity dispersion than DM particles, the $M_{\sigma}$ estimator based on early-type galaxies only is biased low.

Our analysis therefore suggests that the distribution of galaxies in projected phase-space can be used to provide reliable estimates of a cluster mass. In order to optimize the mass estimate, early-type galaxies should be preferentially used as tracers, if a virial mass estimate is required. A simpler, more robust and less biased estimate, especially for small data-sets, can however be obtained from an estimate of the velocity dispersion of all galaxies identified as cluster members. However, it is not within the scope of this paper to set up an optimized observational strategy for the determination of cluster masses. The interloper rejection technique adopted in our analysis has been proven to work efficiently, but perhaps there is room for improvement. We will compare different interloper rejection techniques in a forthcoming paper (Girardi et al., in preparation). Further progress is to be expected by increasing the resolution of the simulations to check the stability of the phase-space sampling of galaxies, and by a more realistic galaxy classification, e.g. based on colours.

Acknowledgements. We dedicate this paper to the memory of Daniel Gerbal We thank Gary Mamon, Lauro Moscardini and Bepi Tormen for useful discussion and the referee for her/his useful comments. The simulations were carried out on the IBM-SP4 machine at the "Centro Interuniversitario del Nord-Est 
per il Calcolo Elettronico" (CINECA, Bologna), with CPU time assigned under a INAF/CINECA grant. This work has been partially supported by the PD-51 INFN grant. This research has made use of NASA's Astrophysics Data System Bibliographic Services.

\section{References}

Abazajian, K., Adelman-McCarthy, J. K., Agüeros, M. A., et al. 2005, AJ, 129, 1755

Abell, G. O. 1958, ApJS, 3, 211

Aceves, H., \& Perea, J. 1999, A\&A, 345, 439

Adami, C., Biviano, A., \& Mazure, A. 1998a, A\&A, 331, 439

Adami, C., Mazure, A., Katgert, P., \& Biviano, A. 1998b, A\&A, 336, 63

Adami, C., Mazure, A., Biviano, A., Katgert, P., \& Rhee, G. 1998c, A\&A, 331, 493

Athreya, R. M., Mellier, Y., van Waerbeke, L., et al. 2002, A\&A, 384, 743

Barrena, R., Biviano, A., Ramella, M., Falco, E. E., \& Seitz, S. 2002, A\&A, 386, 816

Bartelmann, M., \& Steinmetz, M. 1996, MNRAS, 283, 431

Beers, T. C., Flynn, K., \& Gebhardt 1990, AJ, 100, 32

Berlind, A. A., Weinberg, D. H., Benson, A. J., et al. 2003, ApJ, 593, 1

Bird, C. 1995, ApJ, 445, L81

Biviano, A. 2000, in Constructing the Universe with Clusters of Galaxies, ed. F. Durret, \& D. Gerbal, CD-Rom, and

http://nedwww.ipac.caltech.edu/level5/Biviano2/frames.html

Biviano, A., \& Girardi, M. 2003, ApJ, 585, 205

Biviano, A., \& Katgert, P. 2004, A\&A, 424, 779

Biviano, A., Girardi, M., Giuricin, G., Mardirossian, F., \& Mezzetti, M. 1992, ApJ, 396, 35

Biviano, A., Girardi, M., Giuricin, G., Mardirossian, F., \& Mezzetti, M. 1993, ApJ, 411, L13

Biviano, A., Katgert, P., Mazure, A., et al. 1997, A\&A, 321, 84

Biviano, A., Katgert, P., Thomas, T., \& Adami, C. 2002, A\&A, 387, 8

Borgani, S., Gardini, A., Girardi, M., \& Gottlober, S. 1997, New A, 2, 119

Borgani, S., Girardi, M., Carlberg, R. G., Yee, H. K. C., \& Ellingson, E. 1999, ApJ, 527, 561

Borgani, S., Murante, G., Springel, V., et al. 2004, MNRAS, 348, 1078

Bradač, M., Erben, T., Schneider, P., et al. 2005, A\&A, 437, 49

Cappi, A., Benoist, C., da Costa, L. N., \& Maurogordato, S. 2003, A\&A, 408, 905

Carlberg, R. G., Yee, H. K. C., \& Ellingson, E. 1997a, ApJ, 478, 462

Carlberg, R. G., Yee, H. K. C., Ellingson, E., et al. 1997b, ApJ, 476, L7

Cen, R. 1997, ApJ, 485, 39 (C97)

Clowe, D., Trentham, N., \& Tonry, J. 2001, A\&A, 369, 16

Clowe, D., De Lucia, G., \& King, L. 2004, MNRAS, 350, 1038

Colín, P., Klypin, A. A., \& Kravtsov, A. V. 2000, ApJ, 539, 561

Cypriano, E. S., Sodré, L. Jr., Kneib, J.-P., \& Campusano, L. E. 2004, ApJ, 613, 95

Czoske, O., Moore, B., Kneib, J.-P., \& Soucail, G. 2002, A\&A, 386, 31

Dalton, G. B., Efstathiou, G., Maddox, S. J., \& Sutherland, W. J. 1992, ApJ, 390, L1

Danese, L., de Zotti, G., \& di Tullio, G. 1980, A\&A, 82, 322

Danese, L., de Zotti, G., Giuricin, G., et al. 1981, ApJ, 244, 777

Demarco, R., Rosati, P., Lidman, C., et al. 2005, A\&A, 432, 381

den Hartog, R., \& Katgert, P. 1996, MNRAS, 279, 349

Diaferio, A., Kauffmann, G., Colberg, J. M., \& White, S. D. M. 1999, MNRAS, 307,537

Diaferio, A., Geller, M. J., \& Rines, K. J. 2005, ApJ, 628, L97

Diemand, J., Moore, B., \& Stadel, J. 2004, MNRAS, 353, 624

Dolag, K., Bartelmann, M., Perrotta, F., et al. 2004, A\&A, 416, 853

Dressler, A. 1980, ApJ, 236, 351

Dressler, A., \& Shectman, S. A. 1988, AJ, 95, 985 (DS)

Dye, S., Taylor, A. N., Thommes, E. M., et al. 2001, MNRAS, 321, 685

Faltenbacher, A., Kravtsov, A. V., Nagai, D., \& Gottlöber, S. 2005, MNRAS, 358,139

Frenk, C., White, S. D. M., Efstathiou, G., \& Davis, M. 1990, ApJ, 351, 10

Frenk, C., Evrard, A. E., White, S. D. M., \& Summers, F. J. 1996, ApJ, 472, 460

Gao, L., De Lucia, G., White, S. D. M., \& Jenkins, A. 2004, MNRAS, 352, L1

Gavazzi, R. 2005, A\&A, 443, 793

Ghigna, S., Moore, B., Governato, F., et al. 1998, MNRAS, 300, 146

Ghigna, S., Moore, B., Governato, F., et al. 2000, ApJ, 544, 616

Girardi, M., \& Biviano, A. 2002, in Merging Processes in Galaxy Clusters, ed. L. Feretti, I. M. Gioia, \& G. Giovannini (Dordrecht: Kluwer), 39
Girardi, M., Biviano, A., Giuricin, G., Mardirossian, F., \& Mezzetti, M. 1993, ApJ, 404, 38

Girardi, M., Fadda, D., Giuricin, G., et al. 1996, ApJ, 457, 61

Girardi, M., Giuricin, G., Mardirossian, F., Mezzetti, M., \& Boschin, W. 1998, $\mathrm{ApJ}, 505,74$

Gladders, M. D., \& Yee, H. K. C. 2005, ApJS, 157, 1

Goto, T. 2005, MNRAS, 359, 1415

Goto, T., Postman, M., Cross, N. J. G., et al. 2005, ApJ, 621, 188

Haardt, F., \& Madau, P. 1996, ApJ, 461, 20

Katgert, P., Mazure, A., Perea, J., et al. 1996, A\&A, 310, 8

Katgert, P., Mazure, A., den Hartog, R., et al. 1998, A\&AS, 129, 399

Katgert, P., Biviano, A., \& Mazure, A. 2004, ApJ, 600, 657

Kay, S. T., Thomas, P. A., Jenkins, A., \& Pearce, F. 2004, MNRAS, 355, 1091

Klypin, A., Gottlöber, S., Kravtsov, A. V., \& Khokhlov, A. M. 1999, ApJ, 516, 530

Koranyi, D. M., Geller, M. J., Mohr, J. J., \& Wegner, G. 1998, AJ, 116, 2108

Limber, D. N., \& Mathews, W. G. 1960, ApJ, 132, 286

Lin, J.-T., Mohr, J. J., \& Stanford, S. A. 2003, ApJ, 591, 749

Łokas, E. L., \& Mamon, G. A. 2003, MNRAS, 343, 401

Łokas, E. L., Wojtak, R., Gottlöber, S., Mamon, G. A., \& Prada, F. 2005 [arXiv: astro-ph/0511723]

Lombardi, M., Rosati, P., Blakeslee, J. P., et al. 2005, ApJ, 623, 42

Lopes, P. A. A., de Carvalho, R. R., Gal, R. R., et al. 2004, AJ, 128, 1017

Lumsden, S. L., Nichol, R. C., Collins, C. A., \& Guzzo, L. 1992, MNRAS, 258,

Lucey, J. R. 1983, MNRAS, 204, 33

Merchán, M. E., \& Zandivarez, A. 2005, ApJ, 630, 759

Metzler, C. A., White, M., \& Loken, C. 2001, ApJ, 547, 560

Metzler, C. A., White, M., Norman, M., \& Loken, C. 1999, ApJ, 520, L9

Miller, C. J., Nichol, R. C., Reichart, D., et al. 2005, AJ, 130, 968

Macciò, A. V., Murante, G., \& Bonometto, S. P. 2003, ApJ, 588, 35

Moss, C., \& Dickens, R. J. 1977, MNRAS, 178, 701

Murante, G., Arnaboldi, M., Gerhard, O., et al. 2004, ApJ, 607, L83

Nagai, D., \& Kravtsov, A. V. 2005, ApJ, 618, 557

Navarro, J. F., Frenk, C. S., \& White, S. D. M. 1997, ApJ, 490, 493 (NFW)

Oguri, M., Takada, M., Umetsu, K., \& Broadhurst, T. 2005, ApJ, 632, 841

Ota, N., Pointecouteau, E., Hattori, M., \& Mitsuda, K. 2004, ApJ, 601, 120

Perea, J., del Olmo, A., \& Moles, M. 1990, A\&A, 237, 319

Pimbblet, K. A., Smail, I., Edge, A. C., et al. 2006, MNRAS, 366, 645

Popesso, P., Biviano, A., Böhringer, H., Romaniello, M., \& Voges, W. 2005, A\&A, 433, 431

Popesso, P., Biviano, A., Böhringer, H., Romaniello, M., \& Voges, W. 2006, A\&A, 445, 29

Ramella, M., Boschin, W., Fadda, D., \& Nonino, M. 2001, A\&A, 368, 776

Ramella, M., Boschin, W., Geller, M. J., Mahdavi, A., \& Rines, K. 2004, AJ, 128,2022

Rasia, E., Tormen, G., \& Moscardini, L. 2004, MNRAS, 351, 237

Reblinsky, K., \& Bartelmann, M. 1999, A\&A, 345, 1 (RB99)

Reed, D., Governato, F., Quinn, T., et al. 2005, MNRAS, 359, 1357

Ricker, P. M., \& Sarazin, C. L. 2001, ApJ, 561, 621

Rines, K., Geller, M. J., Diaferio, A., Kurtz, M. J., \& Jarrett, T. H. 2004, AJ, 128, 1078

Rosati, P., Borgani, S., \& Norman, C. 2002, ARA\&A, 40, 539

Sanchis, T., Łokas, E. L., \& Mamon, G. A. 2004, MNRAS, 347, 1193

Schindler, S., \& Müller, E. 1993, A\&A, 272, 137

Smith, G. P., Smail, I., Kneib, J.-P., et al. 2002, MNRAS, 333, L16

Sodré, L., Jr., Capelato, H. V., Steiner, J. E., \& Mazure, A. 1989, AJ, 97, 1279

Springel, V. 2005, MNRAS, 364, 1105

Springel, V., \& Hernquist, L. 2003, MNRAS, 339, 289

Springel, V., Yoshida, N., \& White, S. D. M. 2001, New A, 6, 79

Stadel, J. G. 2001, Ph.D. Thesis Univ. Washington

The, L. S., \& White, S. D. M. 1986, AJ, 92, 1248

Thomas, T., \& Katgert, P. 2006, A\&A, 446, 19

Tormen, G., Bouchet, F. R., \& White, S. D. M. 1997, MNRAS, 286, 865

van Haarlem, M. P., Frenk, C. S., \& White, S. D. M. 1997, MNRAS, 287, 817 (vH97)

van Kampen, E., \& Katgert, P. 1997, MNRAS, 289, 327

Voit, G. M. 2005, AdSpR, 36, 701

Wambsganss, J., Bode, P., \& Ostriker, J. P. 2004, ApJ, 603, L93

Wambsganss, J., Bode, P., \& Ostriker, J. P. 2005, ApJ, 635, L1

Wu, X.-P. 2000, MNRAS, 316, 299

Yahil, A., \& Vidal, N. V. 1977, ApJ, 214, 347

Yang, X., Mo, H. J., van den Bosch, F., et al. 2005, MNRAS, 362, 711

Zwicky, F. 1933, Helv. Phys. Acta, 6, 110 